\title{
Linear Perturbations of Hyperkähler Metrics
}

\author{
SERGEI ALEXANDROV ${ }^{1}$, BORIS PIOLINE ${ }^{2,3}$, FRANK SAUERESSIG ${ }^{4}$ and \\ STEFAN VANDOREN ${ }^{5}$ \\ ${ }^{1}$ Laboratoire de Physique Théorique et Astroparticules, CNRS UMR 5207, \\ Université Montpellier II, 34095 Montpellier Cedex 05, France. \\ e-mail: alexandrov@lpta.univ-montp2.fr \\ ${ }^{2}$ Laboratoire de Physique Théorique et Hautes Energies, CNRS UMR 7589, \\ Université Pierre et Marie Curie, 4 place Jussieu, 75252 Paris Cedex 05, France. \\ e-mail: pioline@lpthe.jussieu.fr \\ ${ }^{3}$ Laboratoire de Physique Théorique de l'Ecole Normale Supérieure, CNRS UMR 8549, \\ 24 rue Lhomond, 75231 Paris Cedex 05, France \\ ${ }^{4}$ Institut de Physique Théorique, CEA and CNRS URA 2306, 91191 Gif-sur-Yvette, France. \\ e-mail: frank.saueressig@cea.fr \\ ${ }^{5}$ Institute for Theoretical Physics and Spinoza Institute, Utrecht University, Leuvenlaan 4, \\ 3508 TD Utrecht, The Netherlands.e-mail: S.J.G.Vandoren@uu.nl
}

Received: 4 November 2008 / Revised: 4 February 2009 / Accepted: 9 February 2009

Published online: 26 February 2009 - (C) The Author(s) 2009. This article is published with open access at Springerlink.com

\begin{abstract}
We study general linear perturbations of a class of $4 d$ real-dimensional hyperkähler manifolds obtainable by the (generalized) Legendre transform method. Using twistor methods, we show that deformations can be encoded in a set of holomorphic functions of $2 d+1$ variables, as opposed to the functions of $d+1$ variables controlling the unperturbed metric. Such deformations generically break all tri-holomorphic isometries of the unperturbed metric. Geometrically, these functions generate the symplectomorphisms which relate local complex Darboux coordinate systems in different patches of the twistor space. The deformed Kähler potential follows from these data by a Penrose-type transform. As an illustration of our general framework, we determine the leading exponential deviation of the Atiyah-Hitchin manifold away from its negative mass Taub-NUT limit.
\end{abstract}

Mathematics Subject Classification (2000). 53C26， 53C28， 53C 80.

Keywords. hyperkähler geometry, twistor methods, applications to physics.

\section{Introduction}

Hyperkähler (HK) and quaternionic-Kähler (QK) manifolds appear in a variety of important situations in field and string theories, yet their metrics are rarely known in closed form. In this work, we provide a general formalism for describing linear perturbations of a class of $\mathrm{HK}$ manifolds $\mathcal{M}$ obtainable by the (generalized) Legendre transform method [26,30-32]. While our approach uses well-known twistorial techniques developed in the seminal work [26], our explicit results offer a convenient parametrization of deformations of $\mathrm{HK}$ metrics that should be useful 
in many physics applications involving instanton corrections to moduli space metrics. In the forthcoming work [3], we shall extend our methods to describe linear perturbations of QK manifolds related to the above ones by the superconformal quotient construction [17,43], and in [4] we shall use these results to determine the general form of $\mathrm{D}$-instanton corrections to the hypermultiplet moduli space in type II string theory compactifications.

Before turning to geometry, it is worthwhile elaborating on physics motivations. HK (resp. QK) manifolds generally arise as the target space of rigidly (resp. locally) supersymmetric sigma models with eight supercharges [5,9]. Examples of HK manifolds include the classical moduli space of instantons [7], magnetic monopoles [8] and Higgs bundles [27] in four, three- and two-dimensional Yang-Mills theories respectively, the quantum moduli space of $\mathcal{N}=4$ gauge theories in three dimensions [42], or $K 3$ surfaces that appear as part of the target space for type II (resp. heterotic) superstrings with 16 (resp. 8) supercharges. On the other hand, QK manifolds describe the hypermultiplet moduli spaces of type II superstrings compactified on a Calabi-Yau threefold $X$ [13], or heterotic strings compactified on $K 3$ $[6,44]$. This sector of string theory, far less understood than its vector multiplet cousin (easily described by special Kähler geometry), is the prime motivation for the present work.

In many cases, the geometry is well understood near asymptotic infinity, where the underlying physics admits a weakly coupled description. This is the case for the monopole moduli space at large monopole separations, where the metric asymptotes to a higher-dimensional version of negative-mass Taub-NUT space [23], and also for the moduli space of three-dimensional $\mathcal{N}=4$ gauge theories, where the perturbative series truncates at one-loop - in fact, the two moduli spaces are conjecturally identical [42]. In type II string compactifications, the tree-level asymptotic hypermultiplet metric may be obtained from the metric on the vector multiplet moduli space (exactly computable via mirror symmetry) by the $c$-map construction [13,21]. The one-loop correction is determined by the Euler characteristic of $X$, and there are indications that there are no perturbative corrections beyond one loop [36]. On the heterotic side, the hypermultiplet metric is in principle exactly computable in conformal field theory, but is usually known only in the weak curvature regime [44]. In all these cases, the asymptotic geometry is toric, i.e. possesses the required number of commuting isometries to be obtainable from the Legendre transform construction [26,31]. We return to the geometric significance of this class of metrics below.

Away from this weak coupling region, the metric is usually poorly understood, or in some cases known only implicitly. A notable exception is the moduli space of two $S U$ (2) BPS monopoles, the Atiyah-Hitchin (AH) manifold, whose $S U$ (2)invariant metric may be expressed in terms of elliptic functions [8], and obtained by the generalized Legendre transform construction [32] from an $\mathcal{O}(4)$ projective multiplet [30]. In the limit of large monopole separation, the $\mathcal{O}(4)$ multiplet degenerates into the square of an $\mathcal{O}(2)$ multiplet, and the $\mathrm{AH}$ manifold reduces to 
negative-mass Taub-NUT [23]. At finite separation however, exponential corrections break all tri-holomorphic isometries (see, e.g. [25]), although a tri-holomorphic higher rank Killing tensor does remain [12,20]. More generally, a description of the moduli space of $k S U(2)$ monopoles using $\mathcal{O}(2 j)(1 \leq j \leq k-1)$ projective supermultiplets is known [28], but only in a rather implicit form. Determining the leading exponential correction at long distance is one of the possible applications of the techniques developed in this paper.

Physically, corrections to the asymptotic metric correspond to instanton contributions in field or string theory. In quantum field theory, there is a well-defined procedure to compute these corrections in the one- or two-instanton approximation. Using these techniques, the conjectural equality between the monopole and the 3D field theory moduli spaces was verified in a number of cases $[18,19,22]$. In string theory however, the rules of space-time instanton calculus have not been derived from a microscopic Lagrangian, although they may sometimes be inferred indirectly.

For type IIA string theory (or M-theory) compactified on a Calabi-Yau threefold, the general form of instanton corrections to the hypermultiplet moduli space was first investigated in [10]. More recently, the S-duality of type IIB string theory was used to derive instanton corrections to the hypermultiplet moduli space in general type II compactifications on a Calabi-Yau $X$, for a subset of instanton configurations which preserve the toric isometries [37,38]: namely $D(-1), F 1$ and $D 1$ instantons in type IIB, or $D 2$-branes in an appropriate Lagrangian sublattice in $H^{3}(X, \mathbb{Z})$ (i.e. "wrapping A-cycles only", for a suitable choice of symplectic basis of A and B-cycles) in type IIA. Agreement with the analysis of [35] in the conifold limit was demonstrated in [41].

On the other hand, it has remained an open problem to include the D3 and D5 instantons in type IIB (or the D2-branes wrapping B-cycles in type IIA), as well as NS5 instantons in either theory. As the Kähler classes of $X$ (in type IIA, the complex structure) are taken to infinity, these instanton effects are much smaller than the ones already taken into account. Thus, it is natural to treat them as linear perturbations away from the metric found in [37]. This was carried out for the "universal hypermultiplet" in [1] using techniques germane to four-dimensional QK manifolds, and our aim here and in [3] is to develop similar techniques valid in any dimension.

As indicated above, the relevant $4 d$-dimensional $\mathrm{HK}$ metrics in the weak coupling region are obtainable by the generalized Legendre transform method. This construction, first uncovered in the physics literature using projective superspace techniques [31,32], was interpreted mathematically in the language of twistors in $[12,26,30,33] .^{1}$ In particular, is was shown in [12] that such metrics-which we refer to as $\mathcal{O}(2 n)$ HK spaces for brevity - are characterized by the existence of a local Hamiltonian action of a $d$-dimensional abelian $\mathcal{O}(2-2 n)$-twisted group. They

\footnotetext{
${ }^{1}$ We thank M. Roček and U. Lindström for informing us of their upcoming work [33].
} 
can be encoded in a set of holomorphic functions $H^{[i j]}\left(v^{I}, \zeta\right)$ of $d+1$ variables on the twistor space $\mathcal{Z}$ of $\mathcal{M}$ (one such function for each pair of patches $(i j)$ needed to cover $\mathcal{Z}$, subject to consistency conditions on the overlap of three patches, to local gauge transformations and reality conditions). The case $n=1$ corresponds to "toric" HK manifolds with $d$ commuting tri-holomorphic isometries, obtainable by the standard Legendre transform construction [26], based on $\mathcal{O}(2)$ projective supermultiplets. Cases with $n>1$ correspond to $\mathrm{HK}$ geometries (such as the $\mathrm{AH}$ manifold) with a higher rank Killing tensor, obtainable by the generalized Legendre transform method based on $\mathcal{O}(2 n)$ projective supermultiplets [32].

In this work, we provide a framework to describe general linear perturbations of $4 d$ real-dimensional HK manifolds $\mathcal{M}$ obtainable by the generalized Legendre transform, with special emphasis on $\mathcal{O}(2)$ manifolds. By studying the deformations of the holomorphic symplectic structure on the twistor space, we show that deformations of $\mathcal{M}$ preserving the HK property may be encoded in holomorphic functions $H_{(1)}^{[i j]}\left(v^{I}, \mu_{I}, \zeta\right) \quad(I=1, \ldots, d)$ of $2 d+1$ variables. Such perturbations generically break all isometries (or Killing tensors) of the unperturbed geometry. For $\mathcal{O}(2)$ metrics, we provide explicit formulae (3.38), (4.12) for the complex coordinates of the perturbed metric, and we show that the deformation of the Kähler potential (4.27) in any complex structure can be written as a Penrose-type contour integral along the fibers of the projection $\pi: \mathcal{Z} \rightarrow \mathcal{M}$ (in particular, it is a zeroeigenmode of the Laplace-Beltrami operator, as required by the linearization of the Monge-Ampère equation). Deformations of $\mathcal{O}(2 n)$ metrics could be described by a straightforward generalization of our methods. In the process, we also clarify the significance and ambiguity of the contours arising in the Legendre transform construction of $\mathcal{O}(2 n)$ HK manifolds. We do not address possible obstructions at the non-linear level, nor whether these perturbations lead to geodesically complete metrics.

The outline of this paper is as follows. In Section 2, we review the relation between the HK geometry of $\mathcal{M}$ and the holomorphic symplectic geometry of its twistor space $\mathcal{Z}$, and present a general construction of $\mathcal{Z}$ by patching together local Darboux complex coordinate systems, using complex symplectomorphisms as transition functions. In Section 2.4, we parenthetically argue that this provides a natural framework for the holomorphic quantization of $\mathcal{Z}$, with possible applications to topological string theory. In Section 3, we specialize this construction to the case of $\mathcal{O}(2 n)$ manifolds, and show how the standard generalized Legendre transform construction is recovered. In particular, we obtain an important general formula (3.38) for the twistor lines in the $\mathcal{O}(2)$ case. In Section 4, we study general linear perturbations of $\mathcal{O}(2)$ manifolds. Finally, in Section 5 we illustrate our method on the Taub-NUT and AH manifolds. In particular, we give a simple and elegant representation (5.55), of the leading exponential deviation of the AH manifold away from its Taub-NUT limit. In Appendix A, we show by a direct computation that the deformed geometry is still hyperkähler. 


\section{General Framework}

\subsection{TWISTORIAL CONSTRUCTION OF GENERAL HYPERKÄHLER MANIFOLDS}

We start with a brief review of the twistor approach to hyperkähler manifolds (see $[26,39,40]$ for more details). Let $\mathcal{M}$ be a $4 d$ real-dimensional hyperkähler space. $\mathcal{M}$ admits three integrable complex structures $J^{i}(i=1,2,3)$ satisfying the quaternion algebra

$$
J^{i} J^{j}=\epsilon^{i j k} J^{k}-\delta^{i j},
$$

where $\epsilon_{i j k}$ is the totally antisymmetric tensor with $\epsilon_{123}=1$ and $\delta_{i j}$ is the Kronecker delta symbol. The metric $g(X, Y)$ is hermitian with respect to each of the three complex structures, and each of the Kähler forms

$$
\omega^{i}(X, Y)=g\left(J^{i} X, Y\right)=-\omega^{i}(Y, X),
$$

is closed. With respect to the complex structure $J^{3}$, the two-forms $\omega^{+}=-\frac{1}{2}\left(\omega^{1}-\right.$ $\left.\mathrm{i} \omega^{2}\right)$ and $\omega^{-}=-\frac{1}{2}\left(\omega^{1}+\mathrm{i} \omega^{2}\right)$ are holomorphic and anti-holomorphic, respectively. More generally, any linear combination

$$
J(\zeta, \bar{\zeta})=\frac{1-\zeta \bar{\zeta}}{1+\zeta \bar{\zeta}} J^{3}+\frac{\zeta+\bar{\zeta}}{1+\zeta \bar{\zeta}} J^{2}+\mathrm{i} \frac{\zeta-\bar{\zeta}}{1+\zeta \bar{\zeta}} J^{1}
$$

where $\zeta \in \mathbb{C} \cup \infty=\mathbb{C} P^{1}$ (and $\bar{\zeta}$ its complex conjugate) defines a complex structure on $\mathcal{M}$ compatible with the metric $g$. The corresponding Kähler form is

$$
\omega(\zeta, \bar{\zeta})=\frac{1}{1+\zeta \bar{\zeta}}\left[(1-\zeta \bar{\zeta}) \omega^{3}-2 \mathrm{i} \zeta \omega^{+}+2 \mathrm{i} \bar{\zeta} \omega^{-}\right],
$$

while

$$
\Omega(\zeta)=\omega^{+}-i \zeta \omega^{3}+\zeta^{2} \omega^{-},
$$

is a $(2,0)$ form with respect to the complex structure $J(\zeta, \bar{\zeta})$ [26]. The product $\mathcal{Z}=\mathcal{M} \times \mathbb{C} P^{1}$, equipped with the complex structure $J(\zeta, \bar{\zeta})$ on $\mathcal{M}$ times the standard complex structure on $\mathbb{C} P^{1}$, is a holomorphic fiber bundle on $\mathcal{M}$ known as the twistor space of $\mathcal{M}$. It is a trivial fiber bundle topologically, but not holomorphically. We denote by $p: \mathcal{Z} \rightarrow \mathbb{C} P^{1}$ the projection $(m, \zeta) \mapsto \zeta$, and by $T_{F}=\operatorname{Ker}(\mathrm{d} p)$ the tangent bundle along the fibers of this projection. Fixing a point $m$ on the base, the set $\{m\} \times \mathbb{C} P^{1}$ is a holomorphic section of $p$, known as a twistor line.

The parameter $\zeta$ may be viewed as a local complex coordinate around the north pole $\zeta=0$ of the projective line $\mathbb{C} P^{1}$, and $\Omega$ is regular around the north pole (in fact for all finite $\zeta)$. To remind of this fact, we write $\zeta \equiv \zeta^{[0]}, \Omega(\zeta) \equiv \Omega^{[0]}\left(\zeta^{[0]}\right)$. At the south pole $\zeta=\infty$ however, $\Omega(\zeta)$ has a second order pole. It is convenient to reabsorb this divergence by defining

$$
\Omega^{[\infty]}\left(\zeta^{[\infty]}\right) \equiv \zeta^{-2} \Omega(\zeta)=\omega^{-}-\mathrm{i} \zeta^{[\infty]} \omega^{3}+\left(\zeta^{[\infty]}\right)^{2} \omega^{+},
$$


where $\zeta^{[\infty]}=1 / \zeta^{[0]}$ is a local coordinate around the south pole. The new $\Omega^{[\infty]}\left(\zeta^{[\infty]}\right)$ is still a holomorphic two-form in $J(\zeta, \bar{\zeta})$, but it is now regular around the south pole $\zeta^{[\infty]}=0$. Note that the prefactor $\zeta^{-2}$ in (2.6) is the second power of the transition function $f_{\infty 0}=1 / \zeta$ of the line bundle $\mathcal{O}(1)$ over $\mathbb{C} P^{1}$ (see Section 2.2). Thus, $\Omega^{[0]}$ and $\Omega^{[\infty]}$ should be viewed as local trivializations of a global section $\Omega$ of the holomorphic vector bundle $\Lambda^{2} T_{F}^{*}(2)$ [26].

While the procedure just described produces a holomorphic form $\Omega^{[\infty]}$ regular at the south pole, an a priori different procedure is to apply the antipodal map

$$
\tau:(m, \zeta, \bar{\zeta}) \mapsto(m,-1 / \bar{\zeta},-1 / \zeta)
$$

followed by complex conjugation. The reality conditions $\overline{\omega^{+}}=\omega^{-}, \overline{\omega^{3}}=\omega^{3}$ guarantee that the two procedures are in fact equivalent,

$$
\overline{\tau\left(\Omega^{[0]}\right)}=\Omega^{[\infty]} .
$$

This is summarized by saying that the real structure $\tau$ is compatible with the holomorphic section $\Omega$.

Finally, it can be shown that the normal bundle of the twistor lines is isomorphic to $\mathbb{C}^{2 d} \times \mathcal{O}(1)$. The parameter space of the real twistor lines is therefore a manifold of real dimension $4 d$, isomorphic to the hyperkähler manifold $\mathcal{M}$.

To summarize, any $4 d$ real-dimensional hyperkähler manifold leads to a $2 d+1$ dimensional complex manifold $\mathcal{Z}$ such that

(i) $\mathcal{Z}$ is a holomorphic fiber bundle $p: \mathcal{Z} \rightarrow \mathbb{C} P^{1}$ over the projective line,

(ii) the bundle admits a family of holomorphic sections each with normal bundle isomorphic to $\mathbb{C}^{2 d} \otimes \mathcal{O}(1)$,

(iii) there exists a holomorphic section $\Omega$ of $\Lambda^{2} T_{F}^{*}(2)$ defining a symplectic form on each fiber,

(iv) $\mathcal{Z}$ has a real structure $\tau$ compatible with (i), (ii), (iii) and inducing the antipodal map on $\mathbb{C} P^{1}$.

Conversely, it was shown in [26] (Theorem 3.3) that given $\mathcal{Z}, p, \tau$ satisfying (i), (ii), (iii), (iv) above, the parameter space of real sections of $p$ is a $4 d$ real-dimensional manifold with a natural hyperkähler metric for which $\mathcal{Z}$ is the twistor space. Thus, the construction of hyperkähler metrics reduces to the construction of $\mathcal{O}(2)$ twisted holomorphic symplectic spaces. In the rest of this section, we give a general construction of twistor spaces by patching up local Darboux coordinate systems, generalizing the approach in $[26,30]$.

\subsection{LOCAL SECTIONS OF $\mathcal{O}(m)$ ON $\mathbb{C} P^{1}$}

We first recall the construction of the $\mathcal{O}(m)$ line bundles on $\mathbb{C} P^{1}$. Let $\mathcal{U}_{i}, i=$ $1, \ldots, N$ be a set of open disks with a local coordinate $\zeta^{[i]}$. For each pair of 
patches, we choose an $S U(2)$ transformation $e_{i j}$ which maps $\zeta^{[j]}$ to $\zeta^{[i]}$,

$$
\zeta^{[i]}=\frac{\alpha_{i j} \zeta^{[j]}+\beta_{i j}}{-\bar{\beta}_{i j} \zeta^{[j]}+\bar{\alpha}_{i j}}, \quad\left|\alpha_{i j}\right|^{2}+\left|\beta_{i j}\right|^{2}=1 .
$$

Moreover, we demand that these transformations compose properly, $e_{i j} e_{j k}=e_{i k}$. The quantity

$$
f_{i j}\left(\zeta^{[i]}\right)=\bar{\beta}_{i j} \zeta^{[i]}+\alpha_{i j}=\left(-\bar{\beta}_{i j} \zeta^{[j]}+\bar{\alpha}_{i j}\right)^{-1}
$$

satisfies the cocycle condition

$$
f_{i j}\left(\zeta^{[i]}\right) f_{j k}\left(\zeta^{[j]}\right)=f_{i k}\left(\zeta^{[i]}\right), \quad f_{i j}\left(\zeta^{[i]}\right) f_{j i}\left(\zeta^{[j]}\right)=1
$$

and defines the transition function of the $\mathcal{O}(1)$ line bundle on $\mathbb{C} P^{1}$. By definition, holomorphic sections of the line bundle $\mathcal{O}(m)$ are defined by a set of holomorphic functions $s^{[i]}\left(\zeta^{[i]}\right)$ on each patch $\mathcal{U}_{i}$, such that, on the overlap of two patches $\mathcal{U}_{i} \cap \mathcal{U}_{j}$

$$
s^{[i]}\left(\zeta^{[i]}\right)=f_{i j}^{m} s^{[j]}\left(\zeta^{[j]}\right) .
$$

Equivalently, the differential form $s^{[i]}\left(\zeta^{[i]}\right)\left(\mathrm{d} \zeta^{[i]}\right)^{-m / 2}$ is globally well defined. In particular, $\mathcal{O}(-2)$ corresponds to the bundle of holomorphic one-forms, i.e. the canonical bundle. It is easy to see that non-zero holomorphic global sections of $\mathcal{O}(m)$ only exist for $m \geq 0$, and that in each patch, their Taylor expansion around $\zeta^{[i]}=0$ terminates at order $m$. In fact, the Taylor coefficients of a global section of $\mathcal{O}(m)$ form an irreducible representation of $S U(2)$ of dimension $m+1$ (see, e.g. $[3,29])$.

Rather than using the local coordinate systems $\zeta^{[j]}$ on each of the patches $\mathcal{U}_{j}$, it will be useful to single out a coordinate $\zeta=\zeta^{[0]}$ on a particular patch $\mathcal{U}_{0}$ referred to as the "north pole", and extend it to cover the whole Riemann sphere. In this coordinate, the patch $\mathcal{U}_{j}$ is an open disk centered at $\zeta_{j}=\beta_{0 j} / \bar{\alpha}_{0 j}$ (corresponding to $i=0, \zeta^{[j]}=0$ in (2.9)) and the transition function is

$$
f_{0 j}=\bar{\beta}_{0 j}\left(\zeta+1 / \bar{\zeta}_{j}\right)
$$

Note that for a given choice of $\zeta_{j}$, the $S U(2)$ transformations $e_{0 j}$ (and therefore the transition functions $f_{0 j}$ ) are not uniquely determined: different choices correspond to different local trivializations of $\mathcal{O}(1)$. We shall abuse notation and denote by $s^{[j]}(\zeta)$ the local sections $s^{[j]}\left(\zeta^{[j]}\right)$ referring to the coordinate $\zeta^{[j]}$ implicitly. These local sections are defined in an open neighborhood of $\zeta_{j}$, and may be extended analytically to the whole Riemann sphere, provided no branch cuts are encountered.

Finally, for the purposes of defining the real structure, we shall assume that the antipodal map takes each open disk $\mathcal{U}_{i}$ with local coordinate $\zeta^{[i]}$ to an open disk 
$\mathcal{U}_{\bar{l}}$ with local coordinate $\zeta^{[\bar{i}]}=1 / \zeta^{[i]}$ (namely, $\alpha_{i \bar{l}}=0, \beta_{i \bar{l}}=\mathrm{i}$ ). In the plane parameterized by $\zeta$, the patch $\mathcal{U}_{\bar{l}}$ is centered at $\zeta_{\bar{l}}=-1 / \overline{\zeta_{i}}=-\alpha_{0 i} / \bar{\beta}_{0 i}$.

The patch $\mathcal{U}_{\overline{0}} \equiv \mathcal{U}_{\infty}$ will be referred to as the "south pole", and is related to the north pole by the transition function $f_{0 \infty}=\zeta$. Moreover, it is easy to check that the square of the $\mathcal{O}(1)$ transition functions satisfy the reality condition

$$
\overline{\tau\left(f_{i j}^{2}\right)}=f_{\bar{i} \bar{j}}^{2} .
$$

The transition functions themselves have an inherent sign ambiguity, since the $\mathcal{O}(1)$ bundle does not admit any real structure.

\subsection{LOCAL DARBOUX COORDINATES AND TRANSITION FUNCTIONS}

We now give a general construction of twistor spaces, by gluing together patches where the holomorphic section $\Omega$ is locally trivial. Indeed, by a trivial generalization of the Darboux theorem, it is possible to choose local complex coordinates $\left(v_{[i]}^{I}, \mu_{I}^{[i]}, \zeta^{[i]}\right)(I=1, \ldots, d)$ on $\mathcal{Z}$ such that the holomorphic section $\Omega$ is given locally by

$$
\Omega^{[i]}=\mathrm{d} \mu_{I}^{[i]} \wedge \mathrm{d} \nu_{[i]}^{I} .
$$

We assume that this relation is valid in a neighborhood $\hat{\mathcal{U}}_{i}$ of $\mathcal{Z}$, which projects to the open disk $\mathcal{U}_{i}$ around the point $\zeta_{i}$ on $\mathbb{C} P^{1}$. In analogy to standard Hamiltonian mechanics, we refer to $v_{[i]}^{I}$ and $\mu_{I}^{[i]}$ as the "position" and "momentum" coordinates, respectively. Since $\Omega$ is a section of $\Lambda^{2} T_{F}^{*}(2)$, on the overlap $\hat{\mathcal{U}}_{i} \cap \hat{\mathcal{U}}_{j}$, we must require

$$
\Omega^{[i]}=f_{i j}^{2} \Omega^{[j]} \quad \bmod \mathrm{d} \zeta^{[i]} .
$$

Thus, $\left(v_{[i]}^{I}, \mu_{I}^{[i]}\right)$ and $\left(v_{[j]}^{I}, \mu_{I}^{[j]}\right)$ must be related by a $(\zeta$-dependent) symplectomorphism. A convenient representation of such symplectomorphisms, commonly used in Hamiltonian mechanics, is via generating functions $S^{[i j]}$ of the initial "position" and final "momentum" coordinates, whose derivatives yield the final "position" and initial "momentum": 2

$$
\nu_{[j]}^{I}=\partial_{\mu_{I}^{[j]}} S^{[i j]}\left(\nu_{[i]}, \mu^{[j]}, \zeta^{[i]}\right), \quad \mu_{I}^{[i]}=f_{i j}^{2} \partial_{\nu_{[i]}^{I}} S^{[i j]}\left(\nu_{[i]}, \mu^{[j]}, \zeta^{[i]}\right) .
$$

To see that (2.17) preserves the twisted holomorphic symplectic form, note that the differential of $S^{[i j]}$ is equal to the difference of the Liouville one-forms, up to a locally exact one-form,

$$
\mathrm{d} S^{[i j]}=v_{[j]}^{I} \mathrm{~d} \mu_{I}^{[j]}+f_{i j}^{-2} \mu_{I}^{[i]} \mathrm{d} v_{[i]}^{I} \quad \bmod \mathrm{d} \zeta^{[i]} .
$$

\footnotetext{
${ }^{2}$ The affectation of $f_{i j}^{2}$ to the momentum coordinate $\mu_{I}^{[i]}$ is purely conventional at this stage.
} 
Applying an exterior derivative on either sides then proves (2.16). In general, solving for $\left(v_{[i]}^{I}, \mu_{I}^{[i]}\right)$ in terms of $\left(v_{[j]}^{I}, \mu_{I}^{[j]}\right)$ may lead to ambiguities, but we assume that a prescription is given to lift this degeneracy.

A special solution of (2.16) is to assume that $v_{[i]}^{I}$ and $\mu_{I}^{[i]}$ separately transform as sections of $\mathcal{O}(2 n)$ and $\mathcal{O}(2-2 n)$, such that their product transforms as a section of $\mathcal{O}(2)$ :

$$
v_{[j]}^{I}=f_{i j}^{-2 n} v_{[i]}^{I}, \quad \mu_{I}^{[j]}=f_{i j}^{2 n-2} \mu_{I}^{[i]},
$$

corresponding to the generating function

$$
S^{[i j]}\left(v_{[i]}, \mu^{[j]}, \zeta^{[i]}\right)=f_{i j}^{-2 n} v_{[i]}^{I} \mu_{I}^{[j]} .
$$

We shall assume that the generating function (2.20) controls the "microscopic" structure of the twistor space: in other words, given an open covering by patches $\mathcal{U}_{i}$ with non-trivial symplectomorphisms $S^{[i j]}$, we assume that any refinement of the covering to include a new patch $\mathcal{U}_{j} \subset \mathcal{U}_{i}$ is controlled by the trivial gluing function (2.20).

Of course, the transition functions $S^{[i j]}$ are subject to consistency conditions: in particular $S^{[j i]}$ should be the generating function of the inverse of the symplectomorphism generated by $S^{[i j]}$, determined by the Legendre transform of $S^{[i j]}$.

$$
S^{[j i]}\left(v_{[j]}, \mu^{[i]}, \zeta^{[j]}\right)=\left\langle v_{[i]}^{I} \mu_{I}^{[i]}+f_{i j}^{2} v_{[j]}^{I} \mu_{I}^{[j]}-f_{i j}^{2} S^{[i j]}\left(v_{[i]}, \mu^{[j]}, \zeta^{[i]}\right)\right\rangle_{v_{[i]}, \mu^{[j]}} .
$$

Here, $\langle\cdot\rangle_{x}$ denotes the result of extremising with respect to the variable $x$. When several extrema exist, we assume again that an additional prescription is provided. Moreover, the composition of the symplectomorphisms generated by $S^{[i k]}$ and $S^{[k j]}$ should be the symplectomorphism generated by $S^{[i j]}$,

$$
\begin{aligned}
S^{[i j]}\left(\nu_{[i]}, \mu^{[j]}, \zeta^{[i]}\right)=\langle & f_{j k}^{2} S^{[i k]}\left(\nu_{[i]}, \mu^{[k]}, \zeta^{[i]}\right)+S^{[k j]}\left(\nu_{[k]}, \mu^{[j]}, \zeta^{[k]}\right)- \\
& \left.-f_{j k}^{2} v_{[k]}^{I} \mu_{I}^{[k]}\right\rangle_{\nu_{[k]}, \mu^{[k]}} .
\end{aligned}
$$

Finally, the Darboux form (2.15) of the section $\Omega$ on the patch $\mathcal{U}_{i}$ does not fix the coordinates $\left(\nu_{[i]}, \mu^{[i]}\right)$ uniquely: it is still possible to perform a local symplectomorphism $\left(v_{[i]}, \mu^{[i]}\right) \rightarrow\left(\tilde{v}_{[i]}, \tilde{\mu}^{[i]}\right)$ generated by a holomorphic function $T^{[i]}\left(v_{[i]}, \tilde{\mu}^{[i]}, \zeta^{[i]}\right)$, regular in the patch $\hat{\mathcal{U}}_{i}$,

$$
\tilde{v}_{[i]}^{I}=\partial_{\tilde{\mu}_{I}^{[i]}} T^{[i]}\left(v_{[i]}, \tilde{\mu}^{[i]}, \zeta^{[i]}\right), \quad \mu_{I}^{[i]}=\partial_{v_{[i]}^{I}} T^{[i]}\left(v_{[i]}, \tilde{\mu}^{[i]}, \zeta^{[i]}\right) .
$$

Therefore, the set of transition functions $S^{[i j]}$ defines the same holomorphic symplectic space as

$$
\begin{aligned}
S_{T}^{[i j]}\left(\nu_{[i]}, \mu^{[j]}, \zeta^{[i]}\right)= & \left\langle f_{i j}^{-2}\left(T^{[i]}\left(\nu_{[i]}, \tilde{\mu}^{[i]}, \zeta^{[i]}\right)-\tilde{v}_{[i]}^{I} \tilde{\mu}_{I}^{[i]}\right)+S^{[i j]}\left(\tilde{v}_{[i]}, \tilde{\mu}^{[j]}, \zeta^{[i]}\right)-\right. \\
& -\tilde{v}_{[j]}^{I} \tilde{\mu}_{I}^{[j]}+\left.\tilde{T}^{[j]}\left(\tilde{v}_{[j]}, \mu^{[j]}, \zeta^{[j]}\right)\right|_{\tilde{v}_{[i]}, \tilde{\mu}^{[i]}, \tilde{v}_{[j]}, \tilde{\mu}^{[j]}}
\end{aligned}
$$


where $\tilde{T}^{[i]}$ is the generating function of the inverse of the symplectomorphism (2.23), given by a formula similar to (2.21):

$$
\tilde{T}^{[i]}\left(\tilde{v}_{[i]}, \mu^{[i]}, \zeta^{[i]}\right)=\left\langle v_{[i]}^{I} \mu_{I}^{[i]}+\tilde{v}_{[i]}^{I} \tilde{\mu}_{I}^{[i]}-T^{[i]}\left(v_{[i]}, \tilde{\mu}^{[i]}, \zeta^{[i]}\right)\right\rangle_{\nu_{[i]}, \tilde{\mu}^{[i]}} .
$$

To summarize, a holomorphic symplectic manifold $\mathcal{Z}$ covered by $N$ patches $\hat{\mathcal{U}}_{i}$ is uniquely specified by a set of $N-1$ holomorphic functions $S^{[0 i]}$ of $2 d+1$ variables $v^{I}, \mu_{I}, \zeta$, subject to the equivalence relation $S^{[i j]} \simeq S_{T}^{[i j]}$ in (2.24). The rest of the transition functions is determined by the composition and inversion rules (2.22) and (2.21). With this data in hand, one may in principle solve (2.17) for all pairs of overlapping patches, under the assumption that $v_{[i]}^{I}$ and $\mu_{I}^{[i]}$ are regular at $\zeta^{[i]}=0$, and determine all $\nu_{[i]}^{I}$ and $\mu_{I}^{[i]}$ in each patch as functions of $\zeta$ and an a priori unspecified number $d^{\prime}$ of complex parameters.

While it is useful to discuss the complex and real structures separately, it should be noted at this point that the gauge freedom (2.24) may be used to bring the real structure in a standard form,

$$
\overline{\tau\left(v_{[i]}^{I}\right)}=-v_{[\bar{i}]}^{I}, \quad \overline{\tau\left(\mu_{I}^{[i]}\right)}=-\mu_{I}^{[\bar{l}]} .
$$

Here we have assumed that $n$ is integer; cases with half-integer $n$ can be treated similarly, by imposing (in physics parlance, symplectic Majorana) reality conditions on pairs of $\mathcal{O}(2 n)$ multiplets. The reality conditions (2.26) require that the transition functions $S^{[i j]}$ satisfy

$$
\overline{\tau\left(S^{[i j]}\left(v_{[i]}, \mu^{[j]}, \zeta^{[i]}\right)\right)}=S^{[\bar{i} \bar{j}]}\left(v_{[\bar{l}]}, \mu^{[\bar{j}]}, \zeta^{[\bar{l}]}\right) .
$$

In the particular case $j=\bar{l}$, taking into account that $S^{[\bar{i} i]}$ is related to $S^{[i \bar{i}]}$ by (2.21), we see that the reality condition connects the antipodal map of this transition function with its Legendre transform. Taking into account these reality conditions, the $d^{\prime}$ complex parameters of the twistor lines mentioned above thus become $d^{\prime}$ real parameters. The assumption (ii) of the Theorem implies that $d^{\prime}=4 d$, and that the parameter space is in fact the base $\mathcal{M}$ itself. The functions $v_{[i]}^{I}(\zeta)$ and $\mu_{I}^{[i]}(\zeta)$ provide a parametrization of the "twistor lines", i.e. of the fiber over a point $m$ in $\mathcal{M}$ as a rational curve in $\mathcal{Z}$.

Having determined the Darboux coordinates $\left(v_{[i]}^{I}, \mu_{I}^{[i]}\right)$ as functions of $\zeta$ and of the $4 d$ parameters, it is now straightforward to compute the metric: complex coordinates on $\mathcal{M}$ in the complex structure $J\left(\zeta_{i}, \bar{\zeta}_{i}\right)$ are given by evaluating the twistor lines $\left(v_{[i]}^{I}, \mu_{I}^{[i]}\right)$ at $\zeta^{[i]}=0$ (or equivalently $\zeta=\zeta_{i}$ ),

$$
v_{[i]}^{I}=v_{[i]}^{I}\left(\zeta_{i}\right), \quad w_{I}^{[i]}=\mu_{I}^{[i]}\left(\zeta_{i}\right),
$$

while the Kähler form $\omega\left(\zeta_{i}, \bar{\zeta}_{i}\right) \equiv \omega^{3[i]}$ is given by Taylor expanding the holomorphic section $\Omega^{[i]}$ around $\zeta^{[i]}=0$ :

$$
\Omega^{[i]}\left(\zeta^{[i]}\right)=\mathrm{d} w_{I}^{[i]} \wedge \mathrm{d} v_{[i]}^{I}-\mathrm{i} \omega^{3[i]} \zeta^{[i]}+\mathrm{d} \bar{w}_{I}^{[i]} \wedge \mathrm{d} \bar{v}_{[i]}^{I}\left(\zeta^{[i]}\right)^{2} .
$$


Knowing the complex coordinates and the Kähler form, it is then straightforward to obtain the metric. A Kähler potential $K^{[i]}\left(v_{[i]}, \bar{v}_{[i]}, w^{[i]}, \bar{w}^{[i]}\right)$ may be computed by integrating the hyperkähler form $\omega^{3[i]}$. It will in general depend on the patch $i$; a notable exception is the case of hyperkähler cones, discussed in [3], where it is possible to find a single "hyperkähler potential" $\chi$ which can serve as a Kähler potential for all complex structures at once.

The prescription given just above only applies to the complex structures corresponding to the marked point $\zeta_{i}$ in the patch $\mathcal{U}_{i}$. The complex structure at a point $\zeta_{j}$ lying in the patch $\mathcal{U}_{i}$, but distinct from all $\zeta_{k}, k=1, \ldots, N$ may be described by refining the open covering and adding a new patch $\mathcal{U}_{j}$ centered at $\zeta_{j}$, as indicated below (2.20).

\subsection{A REMARK ON HOLOMORPHIC QUANTIZATION}

As we have indicated repeatedly, the construction of the twistor space $\mathcal{Z}$ via a set of symplectomorphisms relating local Darboux coordinate systems has strong similarities to classical Hamiltonian mechanics, albeit in a holomorphic setting. It is tempting to ask whether this classical construction can be quantized. The following construction naturally suggests itself: to any patch $\mathcal{U}_{i}$, associate a Hilbert space $\mathcal{H}^{[i]}$ of holomorphic functions $\Psi^{[i]}\left(v_{[i]}^{I}, \zeta^{[i]}\right)$. On the overlap $\hat{\mathcal{U}}_{i} \cap \hat{\mathcal{U}}_{j}$, require that

$$
\begin{aligned}
\Psi^{[i]}\left(v_{[i]}, \zeta^{[i]}\right) \sim & f_{i j}^{m} \int \mathrm{d}^{d} \mu_{I}^{[j]} \mathrm{d}^{d} v_{[j]}^{I} \exp \left[\frac{\mathrm{i}}{\hbar}\left(S^{[i j]}\left(v_{[i]}, \mu^{[j]}, \zeta^{[i]}\right)-\mu_{I}^{[j]} v_{[j]}^{I}\right)\right] \times \\
& \times \Psi^{[j]}\left(v_{[j]}, \zeta^{[j]}\right),
\end{aligned}
$$

where $m$ is an undetermined parameter, and $\hbar$ is Planck's constant. Note that for a trivial transition function (2.20), the integral over $\mu_{I}^{[j]}$ imposes the first equation in (2.19) as a delta function, leading to

$$
\Psi^{[i]}\left(v_{[i]}, \zeta^{[i]}\right) \sim f_{i j}^{m} \Psi^{[j]}\left(f_{i j}^{-m} v_{[i]}, \zeta^{[j]}\right)
$$

Thus $\Psi$ is valued in $\mathcal{O}(m)$ within each patch, but may transform non-trivially from one patch to another. The right hand side of (2.30) is the standard semi-classical form of the intertwiner between wave functions in polarizations related by a symplectomorphism generated by $S$, and is only expected to be correct in the limit $\hbar \rightarrow 0$. For finite values of $\hbar$, it should be corrected in order for the intertwiners from $\mathcal{U}_{i}$ to $\mathcal{U}_{j}$ and from $\mathcal{U}_{j}$ to $\mathcal{U}_{k}$ to compose properly. Assuming that this quantum ambiguity can be fixed, (2.30) defines a global Hilbert space $\mathcal{H}_{k}$ which can be viewed as the holomorphic quantization of the space $\mathcal{Z}$. When $\mathcal{M}$ is the Swann bundle $[43,17]$ of the hypermultiplet moduli space, it is tempting to think that $\mathcal{H}_{m}$, for a suitable value of $m$, is the habitat of the generalized topological amplitude postulated in [24]. 


\section{Twistor Spaces of $\mathcal{O}(2 n)$ Hyperkähler Manifolds}

We now restrict to the case of $\mathrm{HK}$ manifolds obtainable by the generalized Legendre transform method [26,32]. As shown in [12], these manifolds are characterized by the fact that they admit a local Hamiltonian action of a $d$-dimensional abelian twistor group (i.e. a Lie group whose parameters are sections of $\mathcal{O}(2-2 n)$ ), or equivalently, by the existence of a covariantly-constant, tri-holomorphic higherrank tensor. For $n=1$, this reduces to the standard Legendre transform construction [26] of $4 d$-dimensional HK manifolds with $d$ commuting tri-holomorphic isometries. The twistorial interpretation of these constructions was explained in [26,30], in a set-up where the twistor space is covered by two patches only, projecting to the north and south pole of $\mathbb{C} P^{1}$. In this section, we extend their analysis to the case of an arbitrary number of patches $\mathcal{U}_{i}$. This extension is needed in order to determine the solutions for the twistor lines (in particular the $\mu_{I}(\zeta)$ ) defined on the entire twistor space $\mathcal{Z}$.

\subsection{SYMPLECTOMORPHISMS COMPATIBLE WITH THE GROUP ACTION}

As explained in [12], the existence of a Hamiltonian action of a $d$-dimensional abelian twistor group implies that there exist $d$ global sections of $\mathcal{O}(2 n)$, corresponding to the moment $\mathrm{map}^{3}$ of these actions. Since these actions commute, we may use them as "position" coordinates $v^{I}$. The fact that they are globally well-defined means that, on $\hat{\mathcal{U}}_{i} \cap \hat{\mathcal{U}}_{j}$,

$$
v_{[j]}^{I}=f_{i j}^{-2 n} \nu_{[i]}^{I} .
$$

In order for (2.16) to hold, the conjugate coordinates $\mu_{I}$ must transform as

$$
\mu_{I}^{[i]}=f_{i j}^{2-2 n} \mu_{I}^{[j]}-f_{i j}^{2} \partial_{v_{[i]}^{I}} \tilde{H}^{[i j]}\left(v_{[i]}, \zeta^{[i]}\right),
$$

where $\tilde{H}^{[i j]}$ may be a function of $\zeta^{[i]}$ and the positions $v_{[i]}^{I}$, but cannot depend on the momenta $\mu_{I}^{[j]}$. This symplectomorphism is of the form (2.17) for a special choice of generating function

$$
S^{[i j]}\left(v_{[i]}, \mu^{[j]}, \zeta^{[i]}\right)=f_{i j}^{-2 n} v_{[i]}^{I} \mu_{I}^{[j]}-\tilde{H}^{[i j]}\left(v_{[i]}, \zeta^{[i]}\right) .
$$

Note that the case $\tilde{H}^{[i j]}=0$ reduces to the transformation rules (2.19). The compatibility constraints (2.21) and (2.22) require that

$$
\tilde{H}^{[j i]}\left(v_{[j]}, \zeta^{[j]}\right)=-f_{i j}^{2} \tilde{H}^{[i j]}\left(v_{[i]}, \zeta^{[i]}\right),
$$

${ }^{3}$ Recall that if $V$ is a Hamiltonian vector field, $\mathcal{L}_{V} \Omega=\left(d l_{V}+l_{V} d\right) \Omega=0$ so $l_{V} \Omega=d \mu_{V}$, where $\mu_{V}$ is the moment map of $V$, defined up to an additive constant. If $V$ is twisted by $\mathcal{O}(2-2 n)$ and $\Omega$ by $\mathcal{O}(2)$, then $\mu_{V}$ is twisted by $\mathcal{O}(2 n)$. 
and

$$
\tilde{H}^{[i k]}\left(v_{[i]}, \zeta^{[i]}\right)+f_{k j}^{2} \tilde{H}^{[k j]}\left(v_{[k]}, \zeta^{[k]}\right)=f_{k j}^{2} \tilde{H}^{[i j]}\left(v_{[i]}, \zeta^{[i]}\right) .
$$

As explained above (2.13), it is convenient to single out the patch $i=0$, and use the complex coordinate $\zeta \equiv \zeta^{[0]}$ as a global coordinate on $\mathcal{Z}$, allowing for poles in the $\zeta$ plane (branch cuts will be discussed in Section 3.4). Moreover, since $v$ is globally well-defined, we may trade the argument $v_{[i]}$ in $\tilde{H}^{[i j]}$ for

$$
\eta^{I}(\zeta) \equiv \zeta^{-n} v_{[0]}^{I}(\zeta)=\sum_{k=-n}^{n} \eta_{k}^{I} \zeta^{k}
$$

and define

$$
H^{[i j]}(\eta, \zeta) \equiv \zeta^{-1} f_{0 j}^{2} \tilde{H}^{[i j]}\left(\zeta^{n} f_{0 i}^{-2 n} \eta, \zeta\right) .
$$

In (3.6) and (3.7), the factors of $\zeta^{-n}$ are conventional, and are inserted to facilitate comparison with earlier studies. In terms of $H^{[i j]}$, the relation (3.2) becomes

$$
\mu_{I}^{[i]}=f_{i j}^{2-2 n} \mu_{I}^{[j]}-\zeta^{1-n} f_{0 i}^{2 n-2} \partial_{\eta^{I}} H^{[i j]}(\eta, \zeta) .
$$

Thanks to the redefinition (3.7), the consistency conditions (2.22) take the simple form

$$
H^{[j i]}=-H^{[i j]}, \quad H^{[i k]}+H^{[k j]}=H^{[i j]},
$$

where all functions depend on the same variables $\eta^{I}$ and $\zeta$. Similarly, the gauge freedom (2.24) with

$$
T^{[i]}\left(v_{[i]}, \tilde{\mu}^{[i]}, \zeta^{[i]}\right)=v_{[i]}^{I} \tilde{\mu}_{I}^{[i]}-\zeta f_{0 i}^{-2} G^{[i]}(\eta, \zeta),
$$

allows to shift

$$
H^{[i j]} \mapsto H^{[i j]}+G^{[i]}-G^{[j]}, \quad \mu_{I}^{[i]} \mapsto \mu_{I}^{[i]}+\zeta^{1-n} f_{0 i}^{2 n-2} \partial_{\eta^{I}} G^{[i]} .
$$

It is important that $\zeta^{1-n} f_{0 i}^{2 n-2} \partial_{\eta^{I}} G^{[i]}$ be a regular function of $\zeta$ in the patch $\hat{\mathcal{U}}_{i}$. Thus, the set of $H^{[i j]}$ should be viewed as a class in the Cech cohomology group $H^{1}(\mathcal{Z})$ valued in the set of $\mu$-independent holomorphic functions. We shall often abuse notation and define $H^{[i j]}$ away from the overlap $\hat{\mathcal{U}}_{i} \cap \hat{\mathcal{U}}_{j}$ (in particular when the two patches do not intersect) using analytic continuation and the second equation in (3.9) to interpolate from $\hat{\mathcal{U}}_{i}$ to $\hat{\mathcal{U}}_{j}$. Ambiguities in the choice of path can be dealt with on a case by case basis.

As in (2.27), reality conditions restrict the possible functions $H^{[i j]}$. The conditions (2.26) on $v^{I}$ translate into

$$
\tau\left(\eta^{I}\right)=(-1)^{n-1} \bar{\eta}^{I} \quad \text { i.e. } \quad \bar{\eta}_{-k}^{I}=(-1)^{n+k-1} \eta_{k}^{I} .
$$


Then, the reality conditions on $\mu_{I}$ require that

$$
\overline{\tau\left(H^{[i j]}\right)}=-H^{[\bar{\imath} \bar{j}]} .
$$

In particular, the transition function relating a patch with its antipodal map must be invariant, $\overline{\tau\left(H^{[i \bar{l}]}\right)}=H^{[i \bar{i}]}$. These conditions ensure that the metric and Kähler potential obtained in the following subsections are real.

\subsection{KÄHLER POTENTIAL AND TWISTOR LINES FOR $\mathcal{O}(2 n)$ MANIFOLDS, $n \geq 2$}

We now proceed to find the twistor lines, i.e. find the most general functions $\eta^{I}(\zeta)$ and $\mu_{I}^{[i]}(\zeta)$ satisfying (3.8), subject to the requirement that the expansion of $\eta^{I}$ in (3.6) has only $2 n+1$ terms, while each $\mu_{I}^{[i]}$ is regular at $\zeta=\zeta_{i}$. According to the Theorem in 2.1 and for suitably generic choices of functions $H^{[i j]}$ obeying hypothesis (ii), the parameter space of these twistor lines will be a $4 d$ dimensional hyperkähler manifold with an action of a $d$-dimensional twistor group. Clearly, the case $n=1$ is quite different from $n \geq 2$ : in the latter case, the number of coefficients $\eta_{k}^{I}$ in (3.6) is greater than $4 d$ and solutions to (3.8) are expected to exist only when $(2 n-3) d$ constraints are imposed on $\eta_{k}^{I}$. In contrast, for $n=1$ the $3 d$ coefficients $\eta_{k}^{I}$ are expected to be unconstrained, and supplemented by $d$ additional parameters coming from the sections $\mu_{I}^{[i]}$ : indeed, for $n=1$ (3.8) is manifestly invariant under global shifts $\mu_{I}^{[i]}(\zeta) \rightarrow \mu_{I}^{[i]}(\zeta)+\varrho_{I}$, corresponding to the $d$ tri-holomorphic isometries. In this subsection, we shall restrict our attention to the case $n \geq 2$, postponing the case $n=1$ to Section 3.3.

Let us consider the Taylor expansion of $\mu_{I}^{[0]}(\zeta)$ around the north pole. The $k$ th coefficient can be obtained from the contour integral

$$
\mu_{I, k}^{[0]}=\frac{1}{2 \pi \mathrm{i}} \oint_{C_{0}} \frac{\mathrm{d} \zeta}{\zeta^{1+k}} \mu_{I}^{[0]}(\zeta)
$$

where $C_{0}$ is a contour around 0 inside $\mathcal{U}_{0}$, oriented counterclockwise. The contour $C_{0}$ may be deformed into a sum of contours inside each $\mathcal{U}_{j}$. Using (3.8) with $i=0$ in each patch, we obtain

$$
\mu_{I, k}^{[0]}=-\frac{1}{2 \pi \mathrm{i}} \sum_{j \neq 0} \oint_{C_{j}} \frac{\mathrm{d} \zeta}{\zeta^{1+k}}\left[f_{0 j}^{2-2 n} \mu_{I}^{[j]}-\zeta^{1-n} \partial_{\eta^{I}} H^{[0 j]}(\eta, \zeta)\right] .
$$

Recall from (2.13) that $f_{0 j}$ has a zero at $\zeta=-1 / \overline{\zeta_{j}}$ and a pole at $\zeta=\infty$. Therefore, for $j \neq \infty$, the first term inside the bracket is regular inside the contour $C_{j}$ and can be omitted. For $j=\infty$, since $f_{0 \infty}=\zeta$, the first term picks the $(2-2 n-k)$ th coefficient in the Taylor expansion of $\mu_{I}^{[\infty]}$ around infinity. Thus, we get

$$
\mu_{I, k}^{[0]}=\mu_{I, 2 n-2-k}^{[\infty]}+\frac{1}{2 \pi \mathrm{i}} \sum_{j} \oint_{C_{j}} \frac{\mathrm{d} \zeta}{\zeta^{k+n}} \partial_{\eta^{I}} H^{[0 j]}(\eta, \zeta),
$$


where the sum over $j$ was trivially extended to include 0 . This equation holds for all values of $k$. However, $\mu_{I, k}^{[0]}$ vanishes if $k<0$, and $\mu_{I, 2 n-2-k}^{[\infty]}$ vanishes if $k>2-2 n$. We conclude that, for $|\ell| \leq n-2$,

$$
\sum_{j} \oint_{C_{j}} \frac{\mathrm{d} \zeta}{2 \pi \mathrm{i} \zeta^{1+\ell}} \partial_{\eta^{I}} H^{[0 j]}(\eta, \zeta)=0
$$

while, for $k \geq 0$,

$$
\begin{aligned}
& \mu_{I, k}^{[0]}=\sum_{j} \oint_{C_{j}} \frac{\mathrm{d} \zeta}{2 \pi \mathrm{i} \zeta^{n+k}} \partial_{\eta^{I}} H^{[0 j]}(\eta, \zeta) \\
& \mu_{I, k}^{[\infty]}=-\sum_{j} \oint_{C_{j}} \frac{\mathrm{d} \zeta}{2 \pi \mathrm{i} \zeta^{2-n-k}} \partial_{\eta^{I}} H^{[0 j]}(\eta, \zeta) .
\end{aligned}
$$

The sections $\mu_{I}^{[0]}$ and $\mu_{I}^{[\infty]}$ may be obtained in terms of the coefficients of $\eta$ by resumming the series,

$$
\begin{aligned}
& \mu_{I}^{[0]}(\zeta)=\sum_{j} \oint_{C_{j}} \frac{\mathrm{d} \zeta^{\prime}}{2 \pi \mathrm{i}\left(\zeta^{\prime}\right)^{n}} \frac{\zeta^{\prime}}{\zeta^{\prime}-\zeta} \partial_{\eta^{I}} H^{[0 j]}(\eta, \zeta), \\
& \mu_{I}^{[\infty]}(\zeta)=\sum_{j} \oint_{C_{j}} \frac{\mathrm{d} \zeta^{\prime}}{2 \pi \mathrm{i}\left(\zeta^{\prime}\right)^{2-n}} \frac{\zeta}{\zeta^{\prime}-\zeta} \partial_{\eta^{I}} H^{[0 j]}(\eta, \zeta),
\end{aligned}
$$

where the first (resp. second) equation holds at the north (resp. south) pole.

On the other hand, the conditions (3.17) give $(2 n-3) d$ constraints on the $(2 n+1) d$ coefficients of the $\eta^{I}$, leaving generally a $4 d$-dimensional set of solutions. These constraints are best described by introducing the "Lagrangian" [31,32]

$$
\mathcal{L}\left(\eta_{k}\right)=\sum_{j} \oint_{C_{j}} \frac{\mathrm{d} \zeta}{2 \pi i \zeta} H^{[0 j]}(\eta, \zeta) .
$$

Note that due to the consistency conditions (3.9), the index 0 on the right-hand side of this expression may be substituted with any other value without changing the result (however, (3.21) is adapted to the complex structure at $\zeta=0$, and to that complex structure only). In terms of $\mathcal{L}$, the constraints (3.17) become

$$
\partial_{\eta_{-k}^{I}} \mathcal{L}=0, \quad|k| \leq n-2,
$$

and in principle allow to eliminate the coefficients $\eta_{-k}^{I},|k| \leq n-2$. The remaining coefficients

$$
v^{I} \equiv \eta_{-n}^{I}, \quad x^{I} \equiv \eta_{1-n}^{I}, \quad \bar{v}^{I} \equiv-\eta_{n}^{I}, \quad \bar{x}^{I} \equiv \eta_{n-1}^{I},
$$

parametrize the twistor lines. The compatibility with the real structure requires that $\bar{v}^{I}$ and $\bar{x}^{I}$ be complex conjugate to $v^{I}, x^{I}$. 
Expanding the holomorphic section $\Omega$ around $\zeta=0$, one easily finds that the holomorphic symplectic form is given by

$$
\omega^{+}=\mathrm{d} w_{I} \wedge \mathrm{d} v^{I},
$$

where

$$
w_{I} \equiv \mu_{I, 0}^{[0]}, \quad \bar{w}_{I} \equiv-\mu_{I, 0}^{[\infty]} .
$$

Thus, $\left(v^{I}, w_{I}\right)$ form a system of holomorphic Darboux coordinates for the complex structure $J^{3}$. The parameters $x^{I}, \bar{x}^{I}$ may be eliminated in favor of $\left(v^{I}, w_{I}\right)$ using (3.18) for $k=0$, which amounts to

$$
\partial_{x^{I}} \mathcal{L}=w_{I} .
$$

To next order in $\zeta$, using

$$
\begin{aligned}
& \mu_{I}^{[0]}(\zeta)=w_{I}+\zeta \partial_{v^{I}} \mathcal{L}+\mathcal{O}\left(\zeta^{2}\right), \\
& \mu_{I}^{[\infty]}(\zeta)=-\bar{w}_{I}+\zeta^{-1} \partial_{\bar{v}^{I}} \mathcal{L}+\mathcal{O}\left(\zeta^{-2}\right),
\end{aligned}
$$

one finds that the Kähler form $\omega^{3}$ descends from a Kähler potential

$$
K\left(v^{I}, \bar{v}^{I}, w_{I}, \bar{w}_{I}\right)=\left\langle\mathcal{L}\left(v^{I}, \bar{v}^{I}, x^{I}, \bar{x}^{I}, \eta_{k}^{I}\right)-x^{I} w_{I}-\bar{x}^{I} \bar{w}_{I}\right\rangle_{x^{I}, \bar{x}^{I}, \eta_{k}^{I}} .
$$

The conditions (3.22) and (3.26) imply that the right-hand side of (3.28) is extremized with respect to $x^{I}, \bar{x}^{I}, \eta_{k}^{I}$, as denoted by the brackets. Thus, the Kähler potential is the Legendre transform of the Lagrangian (3.21) (at vanishing momentum conjugate to $\eta_{k}$ ). The reality conditions (3.13) guarantee that $K$ is real. The complex coordinates and Kähler potential for other choices of complex structure may be obtained by following the same chain of reasoning, but singling out a different "north pole".

\subsection{KÄHLER POTENTIAL AND TWISTOR LINES FOR O(2) MANIFOLDS}

We now turn to the case $n=1$, corresponding to toric HK manifolds with $d$ commuting triholomorphic isometries. As indicated previously, the $\mathcal{O}(2)$ global sections $\eta^{I}$ are unconstrained; we denote their Laurent coefficients at $\zeta=0$ as

$$
\eta^{I}(\zeta)=\frac{v^{I}}{\zeta}+x^{I}-\bar{v}^{I} \zeta
$$

This is the $N=2$ tensor multiplet in projective superspace, as introduced in [31]. On the other hand, local sections $\mu_{I}^{[i]}$ should satisfy (3.8),

$$
\mu_{I}^{[i]}=\mu_{I}^{[j]}-\partial_{\eta^{I}} H^{[i j]}(\eta, \zeta) .
$$

These equations are expected to uniquely determine all the $\mu_{I}^{[i]}$, s (at least locally), up to global shifts $\mu_{I}^{[i]} \rightarrow \mu_{I}^{[i]}+\varrho_{I}$. Indeed, (3.30) can be solved following the same 
steps as in the previous subsection. Setting $n=1$ in (3.16), (3.18) continues to hold for $k>0$. For $k=0$ however, only the difference $\mu_{I, 0}^{[0]}-\mu_{I, 0}^{[\infty]}$ is determined,

$$
\mu_{I, 0}^{[0]}-\mu_{I, 0}^{[\infty]}=\sum_{j} \oint_{C_{j}} \frac{\mathrm{d} \zeta}{2 \pi \mathrm{i} \zeta} \partial_{\eta^{I}} H^{[0 j]}(\eta, \zeta)
$$

Together with $v^{I}, x^{I}, \bar{v}^{I}$, the real combinations

$$
\varrho_{I} \equiv-\mathrm{i}\left(\mu_{I, 0}^{[0]}+\mu_{I, 0}^{[\infty]}\right),
$$

provide the $4 d$ coordinates parametrizing the solution space of (3.30). Defining as before $w_{I} \equiv \mu_{I, 0}^{[0]}, \bar{w}_{I} \equiv-\mu_{I, 0}^{[\infty]}$, we see that $w_{I}+\bar{w}_{I}$ is the parameter conjugate to $x^{I}$ with respect to the Lagrangian (3.21),

$$
w^{I}+\bar{w}^{I}=\partial_{x^{I}} \mathcal{L}, \quad \mathcal{L}\left(v^{I}, \bar{v}^{I}, x^{I}\right)=\sum_{j} \oint_{C_{j}} \frac{\mathrm{d} \zeta}{2 \pi \mathrm{i} \zeta} H^{[0 j]}\left(\eta^{I}, \zeta\right) .
$$

As before, $\left(v^{I}, w_{I}\right)$ provide a system of holomorphic Darboux coordinates on $\mathcal{M}$ in the complex structure $J^{3}$. The holomorphic symplectic form is still given by (3.24), while the Legendre transform of the Lagrangian $\mathcal{L}$ produces a Kähler potential for a hyperkähler metric in this complex structure,

$$
K(v, \bar{v}, w, \bar{w})=\left\langle\mathcal{L}\left(v^{I}, \bar{v}^{I}, x^{I}\right)-x^{I}\left(w_{I}+\bar{w}_{I}\right)\right\rangle_{x^{I}} .
$$

The independence of the Kähler potential on $\mathrm{i} \varrho_{I}=w^{I}-\bar{w}^{I}$ makes it manifest that the metric has $d$ commuting Killing vectors $\partial_{\varrho_{I}}$, which furthermore are tri-holomorphic. The metric on $\mathcal{M}$ may be computed without knowing $x^{I}$ as an explicit function of $\left(v^{I}, \bar{v}_{I}, w_{I}+\bar{w}_{I}\right)$. It is given by [26]

$$
\begin{aligned}
& K_{v^{I} \bar{v}^{J}}=\mathcal{L}_{v^{I} \bar{v}^{J}}-\mathcal{L}_{v^{I} x^{K}} \mathcal{L}^{x^{K} x^{L}} \mathcal{L}_{x^{L} \bar{v}^{J}} \\
& K_{v^{I} \bar{w}_{J}}=\mathcal{L}_{v^{I} x^{K}} \mathcal{L}^{x^{K} x^{J}}, \quad K_{w_{I} \bar{v}^{J}}=\mathcal{L}^{x^{I} x^{K}} \mathcal{L}_{x^{K} \bar{u}^{J}}, \quad K_{w_{I} \bar{w}_{J}}=-\mathcal{L}^{x^{I} x^{J}},
\end{aligned}
$$

where $\mathcal{L}_{x^{I}}=\partial_{x^{I}} \mathcal{L}, \mathcal{L}_{v^{I}}=\partial_{v^{I}} \mathcal{L}$, and $\mathcal{L}^{x^{I} x^{J}}$ denotes the inverse of the matrix $\mathcal{L}_{x^{I} x^{J}}$. The local sections $\mu_{I}^{[0]}$ can be computed by resumming the series (3.18), leading to

$$
\mu_{I}^{[0]}=w_{I}+\frac{\partial}{\partial v^{I}}\left[\sum_{j} \oint_{C_{j}} \frac{\mathrm{d} \zeta^{\prime}}{2 \pi \mathrm{i}} \frac{\zeta H^{[0 j]}\left(\zeta^{\prime}\right)}{\zeta^{\prime}-\zeta}\right],
$$

when $\zeta$ lies inside the north pole patch $\mathcal{U}_{0}$, or

$$
\mu_{I}^{[\infty]}=-\bar{w}_{I}-\frac{\partial}{\partial \bar{v}^{I}}\left[\sum_{j} \oint_{C_{j}} \frac{\mathrm{d} \zeta^{\prime}}{2 \pi \mathrm{i} \zeta^{\prime}} \frac{H^{[0 j]}\left(\zeta^{\prime}\right)}{\zeta^{\prime}-\zeta}\right],
$$


when $\zeta$ lies in the south pole patch $\mathcal{U}_{\infty}$. Inserting $w_{I}=\left(\mathcal{L}_{x^{I}}+\mathrm{i}_{I}\right) / 2$ in (3.36), or $\bar{w}_{I}=\left(\mathcal{L}_{x^{I}}-\mathrm{i}_{I}\right) / 2$ in $(3.37)$, leads to a single expression

$$
\mu_{I}^{[i]}(\zeta)=\frac{\mathrm{i}}{2} \varrho_{I}+\sum_{j} \oint_{C_{j}} \frac{\mathrm{d} \zeta^{\prime}}{2 \pi \mathrm{i} \zeta^{\prime}} \frac{\zeta+\zeta^{\prime}}{2\left(\zeta^{\prime}-\zeta\right)} \partial_{\eta^{I}} H^{[0 j]}\left(\zeta^{\prime}\right),
$$

valid both for $i=0, \zeta \in \mathcal{U}_{0}$ and $i=\infty, \zeta \in \mathcal{U}_{\infty}$. In fact, it is easy to check that (3.38) evaluated for $\zeta \in \mathcal{U}_{i}$ gives the general solution to the matching conditions (3.30). Equation (3.38) is the main new result of this section. It should be noted that the index 0 on the right hand side may be substituted with any other value without changing the result, due to the consistency conditions (3.9). Equations (3.29) and (3.38) provide an explicit parametrization of the twistor lines over any point on $\mathcal{M}$ as rational curves in $\mathcal{Z}$.

\subsection{LOGARITHMIC BRANCH CUTS}

Up till now we assumed that the local coordinates $v_{[i]}(\zeta)$ and $\mu^{[i]}(\zeta)$ could be analytically extended as meromorphic functions on $\mathbb{C} P^{1}$. In many cases of interest however, it is important to relax this assumption and allow for branch cuts. In this subsection we extend the previous construction to the case where the transition function $H^{[i j]}$ contains a singular term $c^{[i j]} \log \eta$, where $\eta$ is one of the $\mathcal{O}(2)$ multiplets $\eta^{I}$ and $c^{[i j]}$ are regular functions of these multiplets. Correspondingly, one expects a singularity in the associated local momentum coordinates $\mu^{[i]}$. An example of this situation is the "improved tensor multiplet" representation of flat $\mathbb{R}^{4}$, discussed in Section 3.5.2 below, and the one-loop corrected hypermultiplet moduli space, to be discussed in [3].

The presence of the logarithmic branch cut gives rise to a Riemann surface which is an infinite cover of $\mathbb{C} P^{1}$. Let us label each sheet by an integer, and single out one particular sheet, say with the label $n$. As before, this sheet can be covered by a set of patches $\mathcal{U}_{i}$, with local Darboux coordinates $v_{[i]}^{I}, \mu_{I}^{[i]}$ satisfying (3.30) on the overlap of two patches. In addition, we must make a choice of branch for $\log \eta$. At this point, it is important to recall that logarithmic branch cuts are oriented: depending whether the angle between the branch cut and the path is positive or negative, one passes from the $n$th sheet to the $n+1$ th or $n-1$ th sheet. We choose the branch cuts for the singular term in $H^{[i j]}$ to extend from $\zeta_{+}$to the origin and from $\zeta_{-}$to infinity, where $\zeta_{ \pm}$are the two zeros of $\eta$ (of course one can flip $\zeta_{+}$ and $\zeta_{-}$).

In this situation, the procedure to solve the equations for $\mu^{[i]}$ presented in Section 3.2 continues to hold, with due attention paid to the cuts. Since $\mu^{[0]}$ is regular at $\zeta=0$, its Taylor coefficients are still given by (3.14). The integral over $C_{0}$ can again be rewritten as a sum of integrals around contours surrounding all $\mathcal{U}_{i}$. Now, however, contours can encounter cuts of $\mu^{[0]}$ and therefore may not be individually closed. For (3.15) to hold, we require that the end point of one 


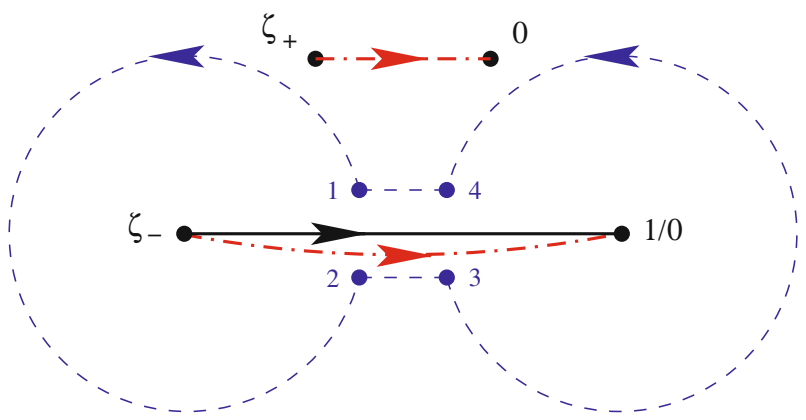

Figure 1. Structure of branch cuts in case (i), $c^{[0-]}=c^{[0 \infty]}$. The solid (black) line represents the branch cut in $\mu^{[0]}(\zeta)$, the semi-dotted (red) line is the branch cut in $H^{[0-]}$, and the dotted (blue) line is the contour $C$ along which $\mu^{[0]}(\zeta)$ is integrated

contour lies on the branch cut, and coincides with the starting point of another contour, in such a way that the sum reproduces a closed contour (see Figure 1). Using (3.15), one may substitute $\mu^{[0]}$ in the patch $\mathcal{U}_{j}$ by $\mu^{[j]}-\partial_{\eta} H^{[0 j]}$ as in (3.30); the first term is regular inside $\mathcal{U}_{j}$, so reproduces the first term in (3.16). The second term can be rewritten as the integral of a holomorphic function on a closed contour, due to additional conditions on the transition functions.

Indeed, let us analyze the possible structure of singularities in $\mu^{[i]}$ and its implications for the coefficients $c^{[i j]}$. The first possibility is that $\mu^{[0]}$ does not have logarithmic singularities. Then they can appear only in the transition functions $H^{[0 j]}$ for $j \neq \pm, \infty$. In this case however, the corresponding contours $C_{j}$ do not cross the branch cuts and therefore can be closed. The second possibility is that $\mu^{[0]}$ does contain branch cut singularities which, under our assumptions, must lie at $\zeta=\zeta_{ \pm}$ and infinity. Since singularities must appear in pairs, the discussion can be reduced to only two cases: (i) $\mu^{[0]}$ has a singular term of the form $\log \left(\zeta-\zeta_{-}\right)$, corresponding to a branch cut from $\zeta_{-}$to $\infty$, or (ii) $\mu^{[0]}$ has a singular term of the form $\log \frac{\zeta-\zeta_{-}}{\zeta-\zeta_{+}}$, corresponding to a branch cut from $\zeta_{-}$to $\zeta_{+}$. Since the addition of the transition function $\partial_{\eta} H^{[0 j]}$ must remove the singularity at $\zeta_{j}$ to produce a multiplet regular in $\mathcal{U}_{j}$, and since $\log \eta \sim \log \left(1-\zeta_{+} / \zeta\right)+\log \left(\zeta-\zeta_{-}\right)$, one concludes that in case (i) one must have $c^{[0 \infty]}=c^{[0-]}$, whereas in the second case one must have $c^{[0+]}=-c^{[0-]} .^{4}$

Let us consider these two possibilities in some more detail. The first situation is shown on Figure 1. The branch cut in $H^{[0-]}$ can be chosen to run from $\zeta_{-}$to $\infty$ and $\zeta_{+}$to 0 . Since we integrate the same function, the two open contours (12) and (34) can be joined into a single closed contour which encircles the cut from $\zeta_{-}$ to $\infty$.

In the second case, the contour along which $\mu^{[0]}(\zeta)$ is integrated initially runs around the cut from $\zeta_{-}$to $\zeta_{+}$. Along the contour (12) (resp. (34)), one may replace

\footnotetext{
${ }^{4}$ Actually, these two cases are related by a gauge transformation (3.11). See the example of the improved tensor multiplet in Section 3.5.2.
} 

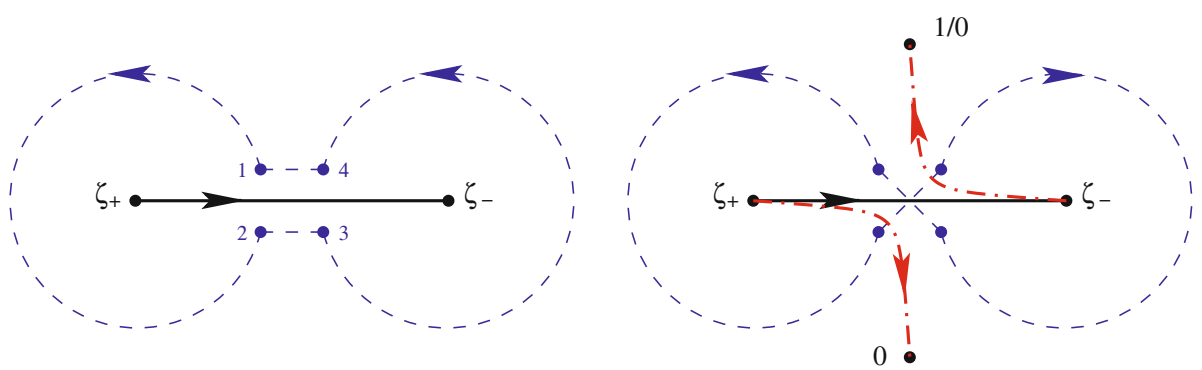

Figure 2. Structure of branch cuts in case (ii), $c^{[0+]}=-c^{[0-]}$. The solid line represents the branch cut in $\mu^{[0]}(\zeta)$, and the semi-dotted line is the branch cut in $H^{[0+]}$. On the left, the dotted line is the contour along which $\mu^{[0]}(\zeta)$ is integrated. On the right, the dotted line is the contour along which the singular part $c^{[0+]} \log \eta$ of $H^{[0+]}$ is integrated. The part of the contour between 2 and 4 lies on the $n+1$ th Riemann sheet of the logarithm

$\mu^{[0]}(\zeta)$ by $\mu^{[+]}-\partial_{\eta}\left(H_{\mathrm{reg}}^{[0+]}+c^{[0+]} \log \eta\right)\left(\right.$ resp. $\left.\mu^{[-]}-\partial_{\eta}\left(H_{\mathrm{reg}}^{[0-]}+c^{[0-]} \log \eta\right)\right)$. The branch cut in $\log \eta$ can be chosen to extend from $\zeta_{+}$to 0 and from $\zeta_{-}$to $\infty$. The contributions from the terms $\mu^{[ \pm]}$drop by the regularity assumption, and the relative negative sign between $c^{[0+]}$ and $c^{[0-]}$ can be eliminated by reversing the contour (34). In the limit where all points 1,2,3,4 coincide, which also implies that the two branch cuts touch each other, it is possible to reconnect the contours (12) and (43) into a single "figure-eight" closed contour, recovering the prescription in [31]. The cuts can then be separated as shown in Figure 2, in such a way that the part of the contour which extends from 2 to 4 crosses into the $n+1$ th Riemann sheet of the logarithm.

The upshot of this discussion is that in cases with logarithmic branch cuts, the same formulae (3.33), (3.38) as in the meromorphic case apply, with the proviso that the integration contours do not just encircle the patches, but encircle or trace a figure-eight contour around the logarithmic branch cuts, depending on the signs of the logarithmic terms $c^{[i j]} \log \eta$ in the transition functions $H^{[i j]}$.

\subsection{EXAMPLE: $\mathbb{R}^{4}$}

We now illustrate the general formalism developed in this section in the simplest case of flat four-dimensional Euclidean space $\mathbb{R}^{4}$. We present two equivalent descriptions, each with its own virtues.

\subsubsection{Free tensor multiplet}

The simplest description of the twistor space of $\mathbb{R}^{4}$ involves a single $\mathcal{O}(2)$ multiplet and two patches around $\zeta=0$ and $\zeta=\infty$, with transition function

$$
H^{[0 \infty]}=\frac{1}{2} \eta^{2}
$$


This is referred to as the "free tensor multiplet" in the physics literature. In order to facilitate the comparison with later subsections, we deviate from the notation in (3.29) and set

$$
\eta=z / \zeta+y-\bar{z} \zeta .
$$

The tensor Lagrangian (3.33) evaluates to

$$
\mathcal{L}(y, z, \bar{z})=-\frac{1}{2} y^{2}+z \bar{z} .
$$

Performing the Legendre transform with respect to $y$ gives a Kähler potential

$$
K=\frac{1}{2}(w+\bar{w})^{2}+z \bar{z}, \quad w+\bar{w}=-y
$$

for the flat metric $\mathrm{d} s^{2}=\mathrm{d} z \mathrm{~d} \bar{z}+\mathrm{d} w \mathrm{~d} \bar{w}$ in the complex structure $J^{3}$. Evaluating (3.36) and (3.37), we obtain

$$
\mu^{[0]}=w+\bar{z} \zeta, \quad \mu^{[\infty]}=-\bar{w}+z / \zeta
$$

consistently with the requirements

$$
\mu^{[\infty]}-\mu^{[0]}=\partial_{\eta} H^{[0 \infty]}, \quad \mu^{[\infty]}=-\overline{\tau\left(\mu^{[0]}\right)} .
$$

Moreover, the holomorphic section $\Omega$ reproduces the correct Kähler forms

$$
\Omega=\zeta \mathrm{d} \mu^{[0]} \wedge \mathrm{d} \eta=\mathrm{d} w \wedge \mathrm{d} z+\zeta(\mathrm{d} \bar{z} \wedge \mathrm{d} z+\mathrm{d} \bar{w} \wedge \mathrm{d} w)+\zeta^{2} \mathrm{~d} \bar{w} \wedge \mathrm{d} \bar{z} .
$$

Observe that $i \zeta \eta$ is the $\mathcal{O}(2)$ valued moment map for the tri-holomorphic isometry $V=i\left(\partial_{w}-\partial_{\bar{w}}\right)$, in the sense that $\mathcal{L}_{V} \Omega=\mathrm{d}(\mathrm{i} \zeta \eta)$.

We now relate this to the standard construction of the twistor space of $\mathbb{R}^{4}$, in [34]. First, observe that despite their linear form, $\mu^{[0]}$ and $\mu^{[\infty]}$ do not patch together into a single $\mathcal{O}(1)$ global section. Rather, the doublet $\left(\mu_{1}^{[0]}, \mu_{1}^{[\infty]}\right)=$ $\left(\mu^{[0]}, \mu^{[0]} / \zeta\right),\left(\mu_{2}^{[0]}, \mu_{2}^{[\infty]}\right)=\left(\zeta \mu^{[\infty]}, \mu^{[\infty]}\right)$ define two different global sections of $\mathcal{O}(1)$, related by a symplectic (Majorana) reality condition $\mu_{A}^{[0]}=\epsilon_{A B} \overline{\tau\left(\mu_{B}^{[\infty]}\right)}$. Note that (3.43) may be rewritten as

$$
\left(\begin{array}{c}
\mu^{[0]} \\
\zeta \mu^{[\infty]}
\end{array}\right)=\left(\begin{array}{cc}
\bar{z} & w \\
-\bar{w} & z
\end{array}\right) \cdot\left(\begin{array}{l}
\zeta \\
1
\end{array}\right)
$$

Defining the doublet $\pi_{[0]}^{A^{\prime}}=(\zeta, 1), \pi_{[\infty]}^{A^{\prime}}=(1,1 / \zeta)$, and $x_{A A^{\prime}}=\left(\begin{array}{cc}\bar{z} & w \\ -\bar{w} & z\end{array}\right)$, Eq. (3.46) becomes

$$
\mu_{A}^{[i]}=x_{A A^{\prime}} \pi_{[i]}^{A^{\prime}}, \quad i=0, \infty .
$$

This reproduces the standard relation between points in $\mathbb{R}^{4}$ and lines in $\mathbb{C} P^{3}$, or two-planes in $\mathbb{C}^{4}$, coordinatized by $\left(\mu_{A}, \pi^{A^{\prime}}\right)$. Unlike the construction in the next subsection, it should be noted that the Kähler potential (3.42) is not invariant under the $S U(2)$ action by right multiplication on the matrix $x_{A A^{\prime}}$. 


\subsubsection{Improved tensor multiplet}

We now describe an equivalent construction of the twistor space of $\mathbb{R}^{4}$, known as the "improved tensor multiplet" in the physics literature [16,31], which makes the superconformal symmetry manifest (see [3] for a discussion of superconformal properties).

Let us introduce four different patches $\mathcal{U}_{0}, \mathcal{U}_{ \pm}, \mathcal{U}_{\infty}$ and the following transition functions

$$
H^{[0+]}=-\frac{1}{2} \eta^{\prime} \log \eta^{\prime}, \quad H^{[0-]}=\frac{1}{2} \eta^{\prime} \log \eta^{\prime}, \quad H^{[0 \infty]}=0,
$$

where $\eta^{\prime}(\zeta)=v / \zeta+x-\bar{v} \zeta$ is a global section of $\mathcal{O}(2)$ (denoted by a prime to avoid confusion with the previous construction). This set of transition functions falls into case (ii) considered in Section 3.4. The Lagrangian for this system is given by the contour integral representation [31]

$$
\mathcal{L}=-\frac{1}{2} \oint_{C} \frac{\mathrm{d} \zeta}{2 \pi \mathrm{i} \zeta} \eta^{\prime} \log \eta^{\prime},
$$

where $C$ is the "figure-eight" contour, encircling the roots $\zeta_{+}$and $\zeta_{-}$of $\eta^{\prime}$,

$$
\zeta_{ \pm}=\frac{x \mp r}{2 \bar{v}}, \quad \zeta_{+} \overline{\zeta_{-}}=-1, \quad \zeta_{+} \zeta_{-}=-v / \bar{v},
$$

counterclockwise and clockwise, respectively, and the radial variable $r$ is defined as

$$
r^{2}=x^{2}+4 v \bar{v} .
$$

The contour integral (3.49) leads to

$$
\mathcal{L}(x, v, \bar{v})=r-x \log \frac{x+r}{2|v|} .
$$

Note that $\mathcal{L}$ is invariant under phase rotations $v \rightarrow \mathrm{e}^{i \theta} v$, and is homogeneous of degree one in $x, v, \bar{v}$. Dualizing $x$ into $u+\bar{u}=\partial_{x} \mathcal{L}$, we find

$$
K=2|v| \cosh (u+\bar{u}), \quad x=-2|v| \sinh (u+\bar{u}) .
$$

Changing variables to

$$
u=\frac{1}{2} \log \frac{w}{z}, \quad v=z w,
$$

leads to the flat space Kähler potential

$$
K=z \bar{z}+w \bar{w} .
$$

Moreover,

$$
\eta^{\prime}=z w / \zeta+(z \bar{z}-w \bar{w})-\bar{z} \bar{w} \zeta
$$


is identified (up to a factor $i \zeta$ ) as the $\mathcal{O}(2)$-valued moment map associated to the rotation $V^{\prime}=i\left(w \partial_{w}-z \partial_{z}-\bar{w} \partial_{\bar{w}}+\bar{z} \partial_{\bar{z}}\right)$.

The twistor line $\mu^{\prime[0]}$ may be computed from (3.36) using the same contour $C$, leading to

$$
\mu^{\prime[0]}=\frac{\mathrm{i}}{2} \varrho-\frac{1}{2} \log \frac{x+\sqrt{x^{2}+4 v \bar{v}}}{2|v|}+\frac{1}{2} \log \frac{1-\zeta / \zeta_{+}}{1-\zeta / \zeta_{-}}=\frac{1}{2} \log \frac{w+\bar{z} \zeta}{z-\bar{w} \zeta} .
$$

The real structure maps $\mu^{\prime[0]}$ to itself, in agreement with the fact that $H^{[0 \infty]}=0$. While $\mu^{\prime[0]}$ is regular around $\zeta=0$ (as it should), it has a logarithmic branch cut on the segment $\left[\zeta_{-}, \zeta_{+}\right]=[z / \bar{w},-w / \bar{z}]$. The logarithmic singularity at $\zeta=\zeta_{+}$cancels from the multiplet

$$
\mu^{\prime[+]}=\mu^{\prime[0]}-\frac{1}{2}\left(\log \eta^{\prime}+1\right),
$$

at the expense of introducing logarithmic singularities at $\zeta_{-}, 0$ and $\infty$. Similarly, $\mu^{\prime[-]}=\mu^{\prime[0]}+\frac{1}{2}\left(\log \eta^{\prime}+1\right)$ is regular at $\zeta_{-}$but has a logarithmic singularities at $\zeta_{+}$, 0 and $\infty$. Altogether, $\mu^{\prime[0]}$ and $\mu^{\prime[ \pm]}$ along with $\eta^{\prime}$ provide regular Darboux coordinates throughout the $\zeta$ plane. They are related to the global $\mathcal{O}(1)$ sections $\mu_{A}$ defined above (3.46) by

$$
\mu_{1}^{[0]}=\sqrt{\zeta} \exp \left(\mu^{[-]}-\frac{1}{2}\right), \quad \mu_{2}^{[0]}=\sqrt{\zeta} \exp \left(-\mu^{\prime[+]}-\frac{1}{2}\right)
$$

whose product is the global $\mathcal{O}(2)$ section $\zeta \eta^{\prime}=\mu_{1}^{[0]} \mu_{2}^{[0]}$. This recovers the standard construction of the twistor space of $\mathbb{R}^{4}$ as the spectral curve $x(\zeta) y(\zeta)=z(\zeta)$ [11]. The relation between the complex Darboux coordinates $\eta^{\prime}, \mu^{\prime}$ and $\eta, \mu$ in the patch $\mathcal{U}_{0}$ is given by the symplectomorphism generated by

$$
S\left(\eta, \mu^{\prime}\right)=\frac{1}{2} \frac{\zeta \eta^{2} \mathrm{e}^{2 \mu^{\prime}}}{1-\zeta \mathrm{e}^{2 \mu^{\prime}}}=\frac{1}{2} \eta \mu, \quad \eta^{\prime}=\mu(\mu+\eta), \quad \mu^{\prime}=\frac{1}{2} \log \frac{\mu}{\zeta(\mu+\eta)} .
$$

In contrast to (3.42), the Kähler potential is invariant under the $S U(2)$ action, and is equal to the hyperkähler potential of $\mathbb{R}^{4}$. As we discuss further in [3], the $S U$ (2) symmetry is realized by fractional linear transformations of $\zeta$. Indeed it is easy to check that

$$
\begin{aligned}
\left(\bar{w} \partial_{z}-\bar{z} \partial_{w}+\partial_{\zeta}\right) v & =0, \\
\left(z \partial_{z}-\bar{z} \partial_{\bar{z}}+w \partial_{w}-\bar{w} \partial_{\bar{w}}+2 \zeta \partial_{\zeta}-2 n\right) v & =0, \\
\left(w \partial_{\bar{z}}-z \partial_{\bar{w}}+\zeta^{2} \partial_{\zeta}-2 n \zeta\right) v & =0,
\end{aligned}
$$

where $(\nu, n)=\left(\zeta \eta^{\prime}, 1\right)$ and $\left(\mu^{\prime}, 0\right)$, respectively.

Finally, we mention that an equivalent description may be obtained by applying the gauge transformation (3.11) to the transition functions (3.48), with

$$
G^{[0]}=\frac{1}{2} \eta^{\prime} \log \left(\eta^{\prime} \zeta\right), \quad G^{[\infty]}=-\frac{1}{2} \eta^{\prime} \log \left(\eta^{\prime} / \zeta\right), \quad G^{[+]}=G^{[-]}=\frac{1}{2} \eta^{\prime} \log \zeta .
$$


This results into a new set of transition functions with

$$
H^{[0+]}=0, \quad H^{[0-]}=H^{[0 \infty]}=\eta^{\prime} \log \eta^{\prime},
$$

representative of case (i) in Section 3.4. The Lagrangian now reads [2]

$$
\mathcal{L}=\oint_{C} \frac{\mathrm{d} \zeta}{2 \pi \mathrm{i} \zeta} H^{[0 \infty]}=\sqrt{x^{2}+4 v \bar{v}}-x-x \log \frac{x+\sqrt{x^{2}+4 v \bar{v}}}{2},
$$

where the contour $C$ encircles the logarithmic branch cut from $\zeta_{-}$to $\infty$. While (3.64) is invariant under a phase rotation of $v$, unlike (3.52) it is not a homogeneous function of $x, v, \bar{v}$. Rather, it satisfies

$$
\left(x \partial_{x}+v \partial_{v}+\bar{v} \partial_{\bar{v}}-1\right) \mathcal{L}=-x .
$$

The occurrence of a linear term in $x$ on the r.h.s. of this equation is consistent with superconformal invariance, we shall discuss it further in [3]. The twistor line $\mu^{\prime[0]}$ following from (3.38) is now given by

$$
\mu^{\prime[0]}=\frac{\mathrm{i}}{2} \varrho^{\prime}-\log \left(1-\frac{\zeta}{\zeta_{-}}\right)-\frac{1}{2}\left(\log \frac{x+r}{2}+1\right) .
$$

In agreement with the general discussion in Section $3.4, \mu^{\prime[0]}$ has logarithmic singularities at $\zeta_{-}$and $\infty$. It can be checked that $\varrho^{\prime}$ in this equation differs from $\varrho$ in (3.57) by $\varrho^{\prime}=\varrho+(1 / 2 i) \log (v / \bar{v})$.

\section{Linear Deformations of $\mathcal{O}(2)$ Hyperkähler Spaces}

In this section, we finally discuss the infinitesimal deformations of $\mathcal{O}(2)$ manifolds (i.e. $4 d$-dimensional $\mathrm{HK}$ manifolds with $d$ commuting tri-holomorphic isometries) which preserve the hyperkähler property but may break all isometries. Our strategy is to perturb the functions $S^{[i j]}$ in (3.3) generating the symplectomorphisms between two patches by arbitrary functions of $v_{[i]}^{I}, \mu_{I}^{[i]}, \zeta^{[i]}$, in a way compatible with the consistency constraints (2.22) and reality conditions (2.27), and work out the corrections to first order in the perturbation. Note that we do not perturb the transition functions $f_{i j}$ of the $\mathcal{O}(1)$ line bundle on $\mathbb{C} P^{1}$, nor the real structure, which can always be chosen as in (2.26) by use of a local symplectomorphism. The same strategy could also be applied to $\mathcal{O}(2 n)$ manifolds with $n \geq 2$, but in view of practical applications we restrict to the case $n=1$.

\subsection{DEFORMING THE SYMPLECTOMORPHISMS}

In line with the strategy just outlined, we consider deformations of the generating functions (3.3) of the following form

$$
S^{[i j]}\left(v_{[i]}, \mu^{[j]}, \zeta^{[i]}\right)=f_{i j}^{-2} v_{[i]}^{I} \mu_{I}^{[j]}-\tilde{H}^{[i j]}\left(v_{[i]}, \zeta^{[i]}\right)-\tilde{H}_{(1)}^{[i j]}\left(v_{[i]}, \mu^{[j]}, \zeta^{[i]}\right),
$$


where $\tilde{H}_{(1)}^{[i j]}\left(\nu_{[i]}, \mu^{[j]}, \zeta^{[i]}\right)$ are functions of $2 d+1$ variables subject to the compatibility constraints to be discussed presently. These functions generate symplectomorphisms

$$
v_{[j]}^{I}=f_{i j}^{-2} v_{[i]}^{I}-\partial_{\mu_{I}^{[j]}} \tilde{H}_{(1)}^{[i j]}, \quad \mu_{I}^{[i]}=\mu_{I}^{[j]}-f_{i j}^{2}\left(\partial_{\nu_{[i]}^{I}} \tilde{H}^{[i j]}+\partial_{v_{[i]}^{I}} \tilde{H}_{(1)}^{[i j]}\right) .
$$

Our task is to determine the linear corrections $\hat{v}_{[i]}^{I}, \hat{\mu}_{I}^{[i]}$ to the unperturbed twistor lines $\breve{v}_{[i]}^{I}, \breve{\mu}_{I}^{[i]}$ such that

$$
v_{[i]}^{I}=\breve{v}_{[i]}^{I}+\hat{v}_{[i]}^{I}, \quad \mu_{I}^{[i]}=\breve{\mu}_{I}^{[i]}+\hat{\mu}_{I}^{[i]},
$$

satisfy (4.2), to linear order in $\tilde{H}_{(1)}^{[i j]}$. Here $\breve{v}_{[i]}^{I}, \breve{\mu}_{I}^{[i]}$ are given by $\breve{v}_{[i]}^{I}=\zeta f_{0 i}^{-2} \eta^{I}$ and (3.38), respectively.

As in (3.7), it is advantageous to express $\tilde{H}_{(1)}^{[i j]}$ in terms of the unperturbed $\mathcal{O}(2)$ global sections $\eta^{I}$ and the local coordinate $\zeta=\zeta^{[0]}$ around the north pole, and define

$$
H_{(1)}^{[i j]}\left(\eta, \breve{\mu}^{[j]}, \zeta\right) \equiv \zeta^{-1} f_{0 j}^{2} \tilde{H}_{(1)}^{[i j]}\left(\zeta f_{0 i}^{-2} \eta, \breve{\mu}^{[j]}, \zeta\right) .
$$

The symplectomorphisms (4.2) are then rewritten as

$$
\begin{aligned}
& \hat{v}_{[j]}^{I}=f_{i j}^{-2} \hat{v}_{[i]}^{I}-\zeta f_{0 j}^{-2} \partial_{\breve{\mu}_{I}^{[j]}} H_{(1)}^{[i j]}, \\
& \hat{\mu}_{I}^{[j]}=\hat{\mu}_{I}^{[i]}+\partial_{\eta^{I}} H_{(1)}^{[i j]}+\zeta^{-1} f_{0 i}^{2} \hat{v}_{[i]}^{J} \partial_{\eta^{I}} \partial_{\eta^{J}} H^{[i j]},
\end{aligned}
$$

while the consistency (2.21), (2.22) and reality conditions (2.27) become

$$
H_{(1)}^{[j i]}\left(\eta, \breve{\mu}^{[i]}, \zeta\right)=-H_{(1)}^{[i j]}\left(\eta, \breve{\mu}^{[j]}, \zeta\right),
$$

together with

$$
H_{(1)}^{[i j]}\left(\eta, \breve{\mu}^{[j]}, \zeta\right)=H_{(1)}^{[i k]}\left(\eta, \breve{\mu}^{[k]}, \zeta\right)+H_{(1)}^{[k j]}\left(\eta, \breve{\mu}^{[j]}, \zeta\right),
$$

and

$$
\overline{\tau\left(H_{(1)}^{[i j]}\right)}=-H_{(1)}^{[\bar{i} \bar{j}]},
$$

respectively. In these expressions, the arguments $\breve{\mu}^{[j]}$ on various patches are to be related to each other using the unperturbed equations (3.30). These conditions can be further simplified by expressing all $\breve{\mu}^{[j]}$ in terms of a single real multiplet ${ }^{5}$

$$
\rho_{I} \equiv-\mathrm{i}\left(\breve{\mu}_{I}^{[0]}+\breve{\mu}_{I}^{[\infty]}\right)=-\mathrm{i}\left(2 \breve{\mu}_{I}^{[i]}+\partial_{\eta^{I}}\left(H^{[i 0]}+H^{[i \infty]}\right)\right) .
$$

${ }^{5}$ Of course, similar multiplets $\rho_{I}^{[i]}(\zeta) \equiv-\mathrm{i}\left(\breve{\mu}_{I}^{[i]}+\breve{\mu}_{I}^{[\bar{i}]}\right)$ can be introduced in each patch; we focus on the one relevant for the complex structure $J^{3}$ at $\zeta=0$. 
Using (3.38), $\rho_{I}$ can be written in the following form

$$
\rho_{I}(\zeta)=\varrho_{I}-\mathrm{i} \sum_{j} \oint_{C_{j}} \frac{\mathrm{d} \zeta^{\prime}}{2 \pi \mathrm{i} \zeta^{\prime}} \frac{\zeta^{\prime}+\zeta}{\zeta^{\prime}-\zeta} \partial_{\eta^{I}} H^{[i j]}\left(\zeta^{\prime}\right)-i \partial_{\eta^{I}}\left(H^{[i 0]}+H^{[i \infty]}\right),
$$

where the pole at $\zeta^{\prime}=\zeta$ is as usual inside $C_{i}$. Similarly to $\eta^{I}$, the multiplet $\rho_{I}$ is manifestly real, $\overline{\tau\left(\rho_{I}\right)}=\rho_{I}$, so the reality conditions (4.8) are automatically obeyed if one requires that $H_{(1)}^{[i \overline{]}]}$ is a real function of $\eta^{I}$ and $\rho_{I}$.

\subsection{DEFORMED TWISTOR LINES}

We now proceed to determine the perturbations of the twistor lines, solving (4.5a) for $\hat{v}_{[i]}^{I}$, substituting the result in $(4.5 \mathrm{~b})$, and finally determine $\hat{\mu}_{I}^{[i]}$. The procedure follows the same steps as in Section 3.2, and we only quote the result. Before this, note however that (4.5) define $\hat{v}_{[i]}^{I}$ and $\hat{\mu}_{I}^{[i]}$ only up to the addition of global $\mathcal{O}(2)$ and $\mathcal{O}(0)$ sections, respectively. Consistently with the reality constraints (2.26), we may fix these ambiguities by imposing

$$
\overline{\hat{v}}_{[0] 1}^{I}=-\hat{v}_{[0] 1}^{I}, \quad \overline{\hat{v}}_{[0] 0}^{I}=\hat{v}_{[0] 2}^{I}, \quad \overline{\hat{\mu}}_{I, 0}^{[0]}=\hat{\mu}_{I, 0}^{[0]} .
$$

In this way, we obtain

$$
\begin{aligned}
& \hat{v}_{[i]}^{I}=\mathrm{i} f_{0 i}^{-2} \sum_{j} \oint_{C_{j}} \frac{\mathrm{d} \zeta^{\prime}}{2 \pi \mathrm{i} \zeta^{\prime}} \frac{\zeta^{3}+\zeta^{\prime 3}}{\zeta^{\prime}\left(\zeta^{\prime}-\zeta\right)} H_{(1)}^{[0 j] I}\left(\zeta^{\prime}\right), \\
& \hat{\mu}_{I}^{[i]}=\sum_{j} \oint_{C_{j}} \frac{\mathrm{d} \zeta^{\prime}}{2 \pi \mathrm{i} \zeta^{\prime}} \frac{\zeta+\zeta^{\prime}}{2\left(\zeta^{\prime}-\zeta\right)} G_{I}^{[0 j]}\left(\zeta^{\prime}\right),
\end{aligned}
$$

where

$$
G_{I}^{[i j]} \equiv H_{(1)}^{[i j]}{ }_{I}+\zeta^{-1} f_{0 i}^{2} \hat{v}_{[i]}^{J} H_{I J}^{[i j]}+\mathrm{i} H_{(1)}^{[i j] J}\left(H_{I J}^{[j 0]}+H_{I J}^{[j \infty]}\right),
$$

and, as usual, $\zeta$ is restricted to lie inside the contour $C_{i}$. Here and below, we use the shorthand notation

$$
H_{I} \equiv \partial_{\eta^{I}} H, \quad H_{I J} \equiv \partial_{\eta^{I}} \partial_{\eta^{J}} H, \quad H_{(1) I} \equiv \partial_{\eta^{I}} H_{(1)}, \quad H_{(1)}^{I} \equiv \partial_{\rho_{I}} H_{(1)}
$$

The second term in (4.13) corresponds to the variation of the unperturbed $\breve{\mu}_{I}^{[i]}$ under $\breve{v}_{[i]}^{I} \rightarrow \breve{v}_{[i]}^{I}+\hat{v}_{[i]}^{I}$, while the third term is due to the change of variable from $\left(v_{[i]}^{I}, \mu_{I}^{[i]}\right)$ to $\left(\eta^{I}, \rho_{I}\right)$. It is easy to verify that $\overline{\tau\left[G_{I}^{[i j]}\right]}=-G_{I}^{[\bar{i} \bar{j}]}$, in accordance with the reality conditions (2.26). 


\subsection{PERTURBED KÄHLER POTENTIAL AND PENROSE TRANSFORM}

Having obtained the deformed twistor lines, the holomorphic 2-form $\omega^{+}$and the Kähler form $\omega^{3}$ in the complex structure $J^{3}$ can be obtained as usual by Taylor expanding the holomorphic section $\Omega^{[0]}=\mathrm{d} \mu_{I}^{[0]} \wedge \mathrm{d} v_{[0]}^{I}$ around $\zeta=0$. The constant term gives

$$
\omega^{+}=\mathrm{d}\left(\frac{\mathrm{i}}{2} \varrho_{I}+\frac{1}{2} \oint_{C} \frac{\mathrm{d} \zeta}{2 \pi \mathrm{i} \zeta}\left(H_{I}+G_{I}\right)\right) \wedge \mathrm{d}\left(v^{I}+\mathrm{i} \oint_{C} \frac{\mathrm{d} \zeta}{2 \pi \mathrm{i}} H_{(1)}^{I}\right),
$$

where, here and henceforth, we suppress the patch indices and denote the sum over contours $C_{i}$ as a single contour $C$. Equation (4.15) identifies

$$
u^{I} \equiv v^{I}+\mathrm{i} \oint_{C} \frac{\mathrm{d} \zeta}{2 \pi \mathrm{i}} H_{(1)}^{I}, \quad w_{I} \equiv \frac{\mathrm{i}}{2} \varrho_{I}+\frac{1}{2} \oint_{C} \frac{\mathrm{d} \zeta}{2 \pi \mathrm{i} \zeta}\left(H_{I}+G_{I}\right)
$$

as holomorphic Darboux coordinates on $\mathcal{M}$. Using (4.10) and (A.3), they can be rewritten as

$$
u^{I}=v^{I}+\mathrm{i} \partial_{\varrho_{I}}\left(\oint_{C} \frac{\mathrm{d} \zeta}{2 \pi \mathrm{i}} H_{(1)}\right), \quad w_{I}=\frac{1}{2}\left(y_{I}+\mathrm{i} \varrho_{I}\right),
$$

where we introduced the real quantity

$$
\begin{aligned}
y_{I}= & \partial_{x^{I}}\left(\oint_{C} \frac{\mathrm{d} \zeta}{2 \pi \mathrm{i} \zeta}\left(H+H_{(1)}\right)\right)- \\
& -\mathrm{i}\left(\oint_{C} \frac{\mathrm{d} \zeta}{2 \pi \mathrm{i} \zeta^{2}} H_{(1)}^{J} \oint_{C} \frac{\mathrm{d} \zeta}{2 \pi \mathrm{i}} H_{I J}-\oint_{C} \frac{\mathrm{d} \zeta}{2 \pi \mathrm{i}} H_{(1)}^{J} \oint_{C} \frac{\mathrm{d} \zeta}{2 \pi \mathrm{i} \zeta^{2}} H_{I J}\right) .
\end{aligned}
$$

On the other hand, the $\mathcal{O}(\zeta)$ term in $\Omega^{[0]}$ leads to the Kähler form

$$
\omega=\mathrm{i}\left[\mathrm{d}\left(\oint_{C} \frac{\mathrm{d} \zeta}{2 \pi \mathrm{i} \zeta^{2}}\left(H_{I}+G_{I}\right)\right) \wedge \mathrm{d} u^{I}+\mathrm{d} w_{I} \wedge \mathrm{d}\left(x^{I}+\mathrm{i} \oint_{C} \frac{\mathrm{d} \zeta}{2 \pi \mathrm{i} \zeta} H_{(1)}^{I}\right)\right] .
$$

The Kähler potential $K(u, \bar{u}, w, \bar{w})$ therefore should satisfy

$$
K_{u^{I}}=\oint_{C} \frac{\mathrm{d} \zeta}{2 \pi \mathrm{i} \zeta^{2}}\left(H_{I}+G_{I}\right), \quad K_{w_{I}}=-x^{I}-\mathrm{i} \oint_{C} \frac{\mathrm{d} \zeta}{2 \pi \mathrm{i} \zeta} H_{(1)}^{I} .
$$

To integrate these equations, it is convenient to introduce the "perturbed Lagrangian"

$$
\mathcal{L}=\oint_{C} \frac{\mathrm{d} \zeta}{2 \pi \mathrm{i} \zeta}\left(H+H_{(1)}\right) \equiv \mathcal{L}_{(0)}+\mathcal{L}_{(1)}
$$


a function of $v^{I}, \bar{v}^{I}, x^{I}, \varrho^{I}$; the derivatives of $\mathcal{L}$ will be denoted by $\mathcal{L}_{x^{I}}=\partial_{x^{I}} \mathcal{L}$, $\mathcal{L}_{v^{I}}=\partial_{v^{I}} \mathcal{L}$, etc. It is also convenient to introduce a special notation for the coefficients

$$
\hat{v}_{0}^{I} \equiv \hat{v}_{[0] 0}^{I}=\oint_{C} \frac{\mathrm{d} \zeta}{2 \pi} H_{(1)}^{I}, \quad \overline{\hat{v}}_{0}^{I} \equiv \overline{\hat{v}}_{[0] 0}^{I}=\oint_{C} \frac{\mathrm{d} \zeta}{2 \pi \zeta^{2}} H_{(1)}^{I} .
$$

Using (A.4) in Appendix A, one can rewrite the complex coordinates as

$$
u^{I}=v^{I}+\hat{v}_{0}^{I}, \quad w_{I}=\frac{1}{2}\left(\mathrm{i} \varrho_{I}+\mathcal{L}_{x^{I}}+\hat{v}_{0}^{J} \mathcal{L}_{v^{J} x^{I}}+\overline{\hat{v}}_{0}^{J} \mathcal{L}_{\bar{v}^{J} x^{I}}\right)
$$

and (4.20) as

$$
K_{u^{I}}=\mathcal{L}_{v^{I}}+\hat{v}_{0}^{J} \mathcal{L}_{v^{J} v^{I}}+\overline{\hat{v}}_{0}^{J} \mathcal{L}_{\bar{v}^{J} v^{I}}, \quad K_{w_{I}}=-\left(x^{I}+\mathrm{i} \mathcal{L}_{\varrho_{I}}\right) .
$$

These two equations can be integrated into

$$
K(u, \bar{u}, w, \bar{w})=\left\langle\mathcal{L}(v, \bar{v}, x, \varrho)+\hat{v}_{0}^{I} \mathcal{L}_{v^{I}}+\overline{\hat{v}}_{0}^{I} \mathcal{L}_{\bar{v}^{I}}-x^{I} y_{I}\right\rangle_{x^{I}} .
$$

Since $u^{I}=v^{I}+\hat{v}_{0}^{I}$, one may rewrite this more concisely as

$$
K(u, \bar{u}, w, \bar{w})=\left\langle\mathcal{L}(u, \bar{u}, x, \varrho)-x^{I}\left(w_{I}+\bar{w}_{I}\right)\right\rangle_{x^{I}} .
$$

Thus, a Kähler potential $K$ for the perturbed HK metric may be obtained by Legendre transform from the perturbed Lagrangian $\mathcal{L}$ defined in (4.21), now considered as a function of $u^{I}, \bar{u}^{I}, x^{I}$ and $\varrho_{I}=-\mathrm{i}\left(w_{I}-\bar{w}_{I}\right)$. In particular, the variation of the Kähler potential is given to first order in the perturbation by

$$
K_{(1)}(u, \bar{u}, w, \bar{w})=\sum_{j} \oint_{C_{j}} \frac{\mathrm{d} \zeta}{2 \pi \mathrm{i} \zeta} H_{(1)}^{[0 j]}(\eta, \rho, \zeta),
$$

where $K_{(1)}(u, \bar{u}, w, \bar{w}) \equiv K(u, \bar{u}, w, \bar{w})-K_{(0)}(u, \bar{u}, w, \bar{w})$, and $K_{(0)}$ is given by the Legendre transform (3.34) of the unperturbed Lagrangian,

$$
K_{(0)}(u, \bar{u}, w, \bar{w})=\left\langle\mathcal{L}_{(0)}\left(u^{I}, \bar{u}^{I}, x^{I}\right)-x^{I}\left(w_{I}+\bar{w}_{I}\right)\right\rangle_{x^{I}} .
$$

while $\eta^{I}$ and $\rho_{I}$ in (4.27) are the unperturbed $\mathcal{O}(2)$ and conjugated multiplet (4.10), evaluated at $\left(x^{I}, u^{I}, \bar{u}^{I}\right)$.

Equation (4.27) is one of the main results of this paper. In words, it says that the variation of the Kähler potential is given by a Penrose-type contour integral of the holomorphic section $H_{(1)}(\eta, \rho, \zeta)$, along the fibers of the projection $\pi: \mathcal{Z} \rightarrow \mathcal{M}$. It is consistent with the fact that $K_{(1)}$ should be a zero-eigenmode of the LaplaceBeltrami operator on $\mathcal{M}$, as required by the linearization of the Monge-Ampère equation. 
As usual, the perturbed metric may be computed without knowing $x^{I}$ as a function of $u^{I}, \bar{u}^{I}, w_{I}, \bar{w}_{I}$ explicitly, using a generalization of (3.35),

$$
\begin{aligned}
& K_{u^{I} \bar{u}^{J}}=\mathcal{L}_{u^{I} \bar{u}^{J}}-\mathcal{L}_{u^{I} x^{K}} \mathcal{L}^{x^{K} x^{L}} \mathcal{L}_{x^{L} \bar{u}^{J}}, \\
& K_{u^{I} \bar{w}_{J}}=\mathcal{L}_{u^{I} x^{K}} \mathcal{L}^{x^{K} x^{J}}+\mathrm{i}\left(\mathcal{L}_{u^{I} \varrho_{J}}-\mathcal{L}_{u^{I} x^{K}} \mathcal{L}^{x^{K} x^{L}} \mathcal{L}_{x^{L} \varrho_{J}}\right),
\end{aligned}
$$

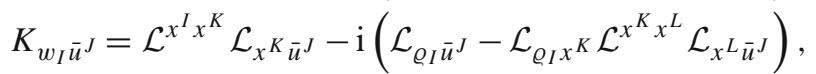

$$
\begin{aligned}
& K_{w_{I} \bar{w}_{J}}=-\mathcal{L}^{x^{I} x^{J}}+\mathcal{L}_{\varrho_{I} \varrho_{J}}+\mathrm{i}\left(\mathcal{L}^{x^{I} x^{K}} \mathcal{L}_{x^{K} \varrho_{J}}-\mathcal{L}_{\varrho_{I} x^{K}} \mathcal{L}^{x^{K} x^{J}}\right) .
\end{aligned}
$$

In Appendix A, we check directly that the deformed metric is hyperkähler, and provided formulae for the inverse metric.

\section{Examples}

We now illustrate our general formalism on two well-known examples, namely Taub-NUT space, which we express as a deformation of flat $\mathbb{R}^{4}$ (preserving one tri-holomorphic isometry), and the AH manifold, which we express as a deformation of Taub-NUT space (breaking all tri-holomorphic isometries).

\subsection{TWISTORIAL DESCRIPTION OF TAUB-NUT SPACE}

The Taub-Nut manifold is described by the superposition of the two Lagrangians (3.41) and (3.52) describing flat $\mathbb{R}^{4}[31,26]$,

$$
\begin{aligned}
\mathcal{L} & =-\frac{1}{2} \oint_{C} \frac{\mathrm{d} \zeta}{2 \pi \mathrm{i} \zeta} \eta \ln \eta-c \oint_{C_{0}} \frac{\mathrm{d} \zeta}{2 \pi \mathrm{i} \zeta} \eta^{2}= \\
& =\left(r-x \ln (r+x)+\frac{1}{2} x \ln (4 v \bar{v})\right)-2 c\left(-\frac{1}{2} x^{2}+v \bar{v}\right),
\end{aligned}
$$

where $c$ is related to the mass parameter as $c=-1 / m$. The Legendre transform of $\mathcal{L}$ leads to the Kähler potential

$$
K=\mathcal{L}-x(u+\bar{u})=r-c\left(x^{2}+2 v \bar{v}\right),
$$

where $x$ is given in terms of $v, \bar{v}, u+\bar{u}$ by the transcendental equation

$$
u+\bar{u}=2 c x-\operatorname{arcsinh}(x / \sqrt{4 v \bar{v}}) .
$$

The derivatives of $K$ can be computed without solving (5.3) explicitly. Using the relations (3.35) for $\mathcal{L}$ being $\varrho$-independent one finds

$$
K_{u \bar{u}}=\frac{r}{1-2 c r}, \quad K_{u \bar{v}}=-\frac{x}{2 \bar{v}(1-2 c r)}, \quad K_{v \bar{v}}=\frac{1-2 c r}{r}+\frac{x^{2}}{4 v \bar{v} r(1-2 c r)} .
$$


Defining spherical coordinates

$$
x=r \cos \theta, \quad v=\frac{1}{2} r \mathrm{e}^{i \phi} \sin \theta, \quad \bar{v}=\frac{1}{2} r \mathrm{e}^{-i \phi} \sin \theta, \quad u-\bar{u}=-i \psi,
$$

the resulting metric takes the usual form,

$$
4 \mathrm{~d} s^{2}=V\left(\mathrm{~d} r^{2}+r^{2} \mathrm{~d} \theta^{2}+r^{2} \sin ^{2} \theta \mathrm{d} \phi^{2}\right)+V^{-1}(\mathrm{~d} \psi+\cos \theta \mathrm{d} \phi)^{2},
$$

where $V$ is a harmonic function in $\mathbb{R}^{3}$

$$
V=\frac{1}{r}-2 c .
$$

For the comparison with the AH manifold in the next section, it is useful to note the exact expression for the local section $\mu$,

$$
\mu^{[0]}(\zeta)=u-2 c \bar{v} \zeta+\frac{1}{2} \ln \left[\frac{1-\zeta / \zeta_{+}}{1-\zeta / \zeta_{-}}\right], \quad \mu^{[\infty]}(\zeta)=-\bar{u}-2 c \frac{v}{\zeta}+\frac{1}{2} \ln \left[\frac{\zeta-\zeta_{+}}{\zeta-\zeta_{-}}\right]
$$

where $u, v$ are related to the "flat space" variables $w, z$ via the coordinate transformation (3.54) and $x$ is a function of $z, w, \bar{z}, \bar{w}$ via (5.3). In terms of the spherical coordinates $r, \theta, \phi, \psi$, the twistor lines become

$$
\begin{aligned}
\eta(\zeta)= & \frac{1}{2 \zeta} r \mathrm{e}^{i \phi} \sin \theta+r \cos \theta-\frac{1}{2} \zeta r \mathrm{e}^{-i \phi} \sin \theta \\
\mu^{[0]}(\zeta)= & -\frac{\mathrm{i}}{2} \psi-\frac{1}{2} \log \cot \frac{\theta}{2}+\frac{1}{2} \log \frac{(\cos \theta+1) \zeta+\mathrm{e}^{\mathrm{i} \phi} \sin \theta}{(\cos \theta-1) \zeta+\mathrm{e}^{\mathrm{i} \phi} \sin \theta}+ \\
& +\operatorname{cr}\left(\cos \theta-\mathrm{e}^{-\mathrm{i} \phi} \sin \theta \zeta\right), \\
\mu^{[\infty]}(\zeta)= & -\frac{\mathrm{i}}{2} \psi+\frac{1}{2} \log \cot \frac{\theta}{2}+\frac{1}{2} \log \frac{\zeta \sin \theta+\mathrm{e}^{\mathrm{i} \phi}(1-\cos \theta)}{\zeta \sin \theta-\mathrm{e}^{\mathrm{i} \phi}(1+\cos \theta)}- \\
& -\operatorname{cr}\left(\cos \theta+\mathrm{e}^{\mathrm{i} \phi} \sin \theta / \zeta\right) .
\end{aligned}
$$

One may check explicitly that any function of $\eta, \mu^{[0]}$ and $\mu^{[\infty]}$ is a zero eigenmode of the Laplace-Beltrami operator $\Delta$ on (5.6). In particular, the Penrose-type contour integral

$$
\oint_{\Gamma} \frac{\mathrm{d} \zeta}{2 \pi i \zeta} H_{(1)}\left(\eta(\zeta), \mu^{[\infty]}(\zeta), \zeta\right),
$$

produces a zero eigenmode of the Laplace-Beltrami operator on Taub-NUT space, for any choice of function $H_{(1)}$ and contour $\Gamma$. In (5.55) below, we identify the function $H_{(1)}$ governing the leading exponential deviation of AH space away from its Taub-NUT limit. 


\subsection{TAUB-NUT AS A DEFORMATION OF $\mathbb{R}^{4}$}

To illustrate our formalism, we exhibit the Taub-Nut space as a deformation of $\mathbb{R}^{4}$, both in the $c \rightarrow 0$ (i.e. short distance $r$ ) regime and the $c \rightarrow \infty$ (long distance) regime. These examples are somewhat trivial, since the perturbation does not break the tri-holomorphic isometry.

In the limit $c \rightarrow 0$ keeping $u, v$ fixed, (5.3) can be solved to first order in $c$,

$$
x=|v|\left(\mathrm{e}^{-(u+\bar{u})}-\mathrm{e}^{u+\bar{u}}\right)+2 c|v|^{2}\left(\mathrm{e}^{-2(u+\bar{u})}-\mathrm{e}^{2(u+\bar{u})}\right)+\mathcal{O}\left(c^{2}\right) .
$$

Substituting in (5.2), this leads to

$$
K=|v|\left(\mathrm{e}^{-(u+\bar{u})}+\mathrm{e}^{u+\bar{u}}\right)+c|v|^{2}\left(\mathrm{e}^{-2(u+\bar{u})}+\mathrm{e}^{2(u+\bar{u})}-4\right)+\mathcal{O}\left(c^{2}\right) .
$$

Performing the coordinate transformation (3.54) this becomes

$$
K=z \bar{z}+w \bar{w}+c\left(z^{2} \bar{z}^{2}+w^{2} \bar{w}^{2}-4 z \bar{z} w \bar{w}\right)+\mathcal{O}\left(c^{2}\right) .
$$

Note that the first order variation of $K$ is equal to the Lagrangian (3.41), evaluated at the point (3.56). This indeed agrees with (4.27) for a linear perturbation with generating function

$$
\tilde{H}_{(1)}(\eta, \mu, \zeta)=-c \eta^{2} .
$$

Moreover, it is easy to check that the first order corrections to the sections $\eta$ and $\mu$ are also consistent with (4.12),

$$
\begin{aligned}
\eta(\zeta) & =\frac{w z}{\zeta}+(z \bar{z}-w \bar{w})-\bar{w} \bar{z} \zeta+2 c\left((z \bar{z})^{2}-(w \bar{w})^{2}\right), \\
\mu^{[0]}(\zeta) & =\frac{1}{2} \ln \left[\frac{w+\bar{z} \zeta}{z-\bar{w} \zeta}\right]-c \zeta\left[2 \bar{w} \bar{z}-\frac{(w \bar{w}-z \bar{z})(w \bar{w}-z \bar{z}+2 \bar{w} \bar{z} \zeta)}{(z-\bar{w} \zeta)(w+\bar{z} \zeta)}\right], \\
\mu^{[\infty]}(\zeta) & =\frac{1}{2} \ln \left[\frac{w+\bar{z} \zeta}{z-\bar{w} \zeta}\right]+\frac{i \pi}{2}-c\left[\frac{2 w z}{\zeta}+\frac{(w \bar{w}-z \bar{z})(\zeta(w \bar{w}-z \bar{z})-2 w z)}{(z-\bar{w} \zeta)(w+\bar{z} \zeta)}\right] .
\end{aligned}
$$

In the opposite regime $c \rightarrow \infty$, corresponding to distances $r \gg 1 / c$, it is appropriate to redefine $u=-2 c w, v=z, x=y$. Expanding (5.3) at large $c$ with fixed $z, w$, one obtains

$$
y=-(w+\bar{w})-\frac{1}{2 c} \operatorname{arcsinh}\left(\frac{w+\bar{w}}{2|z|}\right)+\mathcal{O}\left(c^{-2}\right) .
$$

Substituting into (5.2) leads to

$$
\begin{aligned}
K= & -2 c\left[z \bar{z}+\frac{1}{2}(w+\bar{w})^{2}\right]+\left[\sqrt{(w+\bar{w})^{2}+4 z \bar{z}}-(w+\bar{w}) \operatorname{arcsinh}\left(\frac{w+\bar{w}}{2|z|}\right)\right]+ \\
& +\mathcal{O}\left(c^{-1}\right)
\end{aligned}
$$


Up to an overall factor $-2 c$, the first term is equal to the Kähler potential of flat space (3.42), while the first order correction reproduces the Lagrangian (3.52). This is consistent with (4.27), for a linear perturbation with generating function

$$
\tilde{H}_{(1)}(\eta, \mu, \zeta)=-\frac{1}{2} \eta \log \eta,
$$

integrated along the standard "figure-eight" contour.

\subsection{ATIYAH-HITCHIN AS A DEFORMATION OF TAUB-NUT}

The $\mathcal{O}(4)$ tensor multiplet formulation of the AH space was given in [30] and further analyzed in [29], to which we refer for additional details. In this section, we compute the leading exponential correction away from the Taub-NUT limit, and cast it into our formalism.

\subsubsection{Atiyah-Hitchin as an $\mathcal{O}(4)$ manifold}

As shown in [29,30], the AH manifold can be obtained by the generalized Legendre transform method from the Lagrangian

$$
\mathcal{L}=2 \omega \oint_{\Gamma_{0}} \frac{\mathrm{d} \zeta}{2 \pi \mathrm{i} \zeta} \eta^{(4)}-\oint_{\Gamma} \frac{\mathrm{d} \zeta}{\zeta} \sqrt{\eta^{(4)}},
$$

where $\Gamma_{0}$ encircles $\zeta=0$ while $\Gamma$ winds once around the branch cut between the roots $\alpha$ and $-1 / \bar{\beta}$ of the $\mathcal{O}(4)$ tensor multiplet ${ }^{6}$

$$
\eta^{(4)}=\frac{z}{\zeta^{2}}+\frac{w}{\zeta}+y-\bar{w} \zeta+\bar{z} \zeta^{2}=\frac{\rho}{\zeta^{2}} \frac{(\zeta-\alpha)(\bar{\alpha} \zeta+1)}{1+\alpha \bar{\alpha}} \frac{(\zeta-\beta)(\bar{\beta} \zeta+1)}{1+\beta \bar{\beta}} .
$$

In this subsection we recall how the coefficients of $\eta^{(4)}$ as well as the Kähler potential can be expressed in terms of elliptic functions, which will provide a convenient starting point for extracting the Taub-NUT limit.

The section $\eta^{(4)}$ defines a real algebraic elliptic curve, given by a two-sheeted covering of the $\zeta$ plane with branch points as $\zeta \in\{\alpha,-1 / \bar{\alpha}, \beta,-1 / \bar{\beta}\}$. By a change of coordinates, the curve can be brought into Weierstrass normal form

$$
Y^{2}=X^{3}-g_{2} X-g_{3},
$$

where the invariants $g_{2}, g_{3}$ are given by

$$
\begin{aligned}
& g_{2}=y_{+}^{2}+y_{+} y_{-}+y_{-}^{2}+\frac{1}{4}\left(y_{+}-y_{-}\right)\left(w_{-}^{2}+w_{+}^{2}\right), \\
& g_{3}=-\left(y_{+}+y_{-}\right) y_{+} y_{-}-\frac{1}{4}\left(y_{+}-y_{-}\right)\left(y_{+} w_{-}^{2}+y_{-} w_{+}^{2}\right),
\end{aligned}
$$

${ }^{6}$ Note that we change notations $x \rightarrow y, v \rightarrow \bar{w}, z \rightarrow \bar{z}, x_{ \pm} \rightarrow y_{ \pm}, v_{ \pm} \rightarrow w_{ \pm}, r^{\prime} \rightarrow r$ with respect to [29], to conform to the notations of the present paper. The variable $v_{ \pm}$defined below should not be confused with the namesake variable in [29]. 
with

$$
y_{ \pm}=\frac{y \pm 6|z|}{3}, \quad w_{-}=\operatorname{Re} \frac{w}{\sqrt{z}}, \quad w_{-}=\operatorname{Im} \frac{w}{\sqrt{z}} .
$$

The tensor coordinates $z, w, y, \bar{w}, \bar{z}$ determine four points in the $(X, Y)$ plane,

$$
\left(y_{-}, \pm w_{-}\left(y_{+}-y_{-}\right) / 2\right), \quad\left(y_{+}, \pm \mathrm{i} w_{+}\left(y_{+}-y_{-}\right) / 2\right) .
$$

The curve (5.21) may be uniformized by a parameter $u \in \mathbb{C} /\left(2 \omega \mathbb{Z}+2 \omega^{\prime} \mathbb{Z}\right)$, where $\omega$ and $\omega^{\prime}$ are the half-periods, using the Weierstrass function $\mathcal{P}\left(u, 4 g_{2}, 4 g_{3}\right) \sim 1 / u^{2}+$ $\mathcal{O}\left(u^{2}\right)$,

$$
X=\mathcal{P}\left(u ; 4 g_{2}, 4 g_{3}\right), \quad Y= \pm \frac{1}{2} \mathcal{P}^{\prime}\left(u ; 4 g_{2}, 4 g_{3}\right),
$$

where the prime denotes a $u$ derivative. The Weierstrass function may in turn be represented in terms of Jacobi theta functions,

$$
\mathcal{P}\left(u ; 4 g_{2}, 4 g_{3}\right)=\left(\frac{\pi}{2 \omega}\right)^{2}\left[\left(\frac{\vartheta_{2}(0, \tau) \vartheta_{3}(0, \tau) \vartheta_{4}(v, \tau)}{\vartheta_{1}(v, \tau)}\right)^{2}-\frac{\vartheta_{3}^{4}(0, \tau)+\vartheta_{2}^{4}(0, \tau)}{3}\right],
$$

where the $u$ variable on the 1.h.s. is related to the variable $v$ on the r.h.s. by $v=$ $\pi u /(2 \omega)$, and

$$
\vartheta_{1}(v, \tau)=2 q^{1 / 4} \sum_{n=0}^{\infty}(-1)^{n} q^{n(n+1)} \sin [(2 n+1) v], \text { etc. }
$$

Note that we use the nome $q=\mathrm{e}^{\pi \mathrm{i} \tau}$ instead of the more commonly used modular parameter $\mathrm{e}^{2 \pi \mathrm{i} \tau}$. The modular parameter is $\tau=\omega^{\prime} / \omega$. We note that the half-period is set to a constant $\omega$, which sets the scale of the Taub-NUT space, while the half-period $\omega^{\prime}$, and hence $\tau$, are purely imaginary. We abuse notation and write $\mathcal{P}(v)=\mathcal{P}\left(u ; 4 g_{2}, 4 g_{3}\right)$.

The invariants $g_{2}$ and $g_{3}$ may be expressed in terms of the normalized Eisenstein series $E_{4}=1+240 q^{2}+\cdots$ and $E_{6}=1-504 q^{2}+\cdots$ via

$$
g_{2}=\frac{1}{3}\left(\frac{\pi}{2 \omega}\right)^{4} E_{4}, \quad g_{3}=\frac{2}{27}\left(\frac{\pi}{2 \omega}\right)^{6} E_{6},
$$

Having expressed the uniformizing variable $v=\pi u /(2 \omega)$ in terms of $X$, we define the elliptic integral of the third kind [29]

$$
\pi(v)=-\frac{\pi}{2} \frac{\vartheta_{1}^{\prime}(v, \tau)}{\vartheta_{1}(v, \tau)} \equiv \pi(X),
$$

where the prime is now a $v$ derivative, and the last equality is an abuse of notation. In particular $\pi\left(y_{+}\right)$is imaginary, while $\pi\left(y_{-}\right)$is real. 
The real $\mathcal{O}(4)$ twistor lines are given by enforcing one condition which expresses the $x$ coordinate in (5.20) in terms of $z, v, \bar{z}, \bar{v}$. Moreover, complex coordinates $U, Z, \bar{U}, \bar{Z}$ can be obtained by solving

$$
U=\pi\left(y_{-}\right)+\pi\left(y_{+}\right), \quad \bar{U}=\pi\left(y_{-}\right)-\pi\left(y_{+}\right) .
$$

The coordinates $U, Z$ are related to the coordinates $u, z$ appearing in the Legendre transform construction by

$$
U=u \sqrt{z}, \quad Z=2 \sqrt{z} .
$$

Here $u$ is conjugate to $v$, and should not be confused with the argument of the Weierstrass function.

Equation (5.31) allows to express $y_{ \pm}$, and therefore $x$ and $|Z|$, in terms of $U, \bar{U}$ and $\tau$. In particular, we can compute the elliptic modulus $\tau$ in terms of $U, \bar{U}$ and $|Z|$, and plug into the expression for $x$. The variables $v_{ \pm}$, and therefore $v$ and $\bar{v}$, can be computed from the fact that (5.25) lie on the curve. The Kähler potential in the complex structure where $U, Z$ are complex coordinates is given by

$$
K=4 \eta-\left(y_{+}+y_{-}\right) \omega,
$$

where $\eta$ is the Weierstrass function

$$
\eta=-\frac{\pi^{2}}{12 \omega} \frac{\vartheta_{1}^{\prime \prime \prime}(0, \tau)}{\vartheta_{1}^{\prime}(0, \tau)}=\frac{\pi^{2}}{12 \omega} E_{2}(\tau)
$$

with $E_{2}=1-24 q^{2}+\cdots$ the almost-modular Eisenstein series of weight 2 .

\subsubsection{Strict Taub-NUT limit}

We now extract the limit of the AH manifold as $\tau \rightarrow 0$. To study this limit, we perform a modular transformation to $\tau^{\prime}=-1 / \tau$. In the strict limit $\tau=0$, the elliptic curve (5.20) factorizes as the square of a rational curve,

$$
\eta^{(4)}=\left(\eta^{(2)}\right)^{2}=\left(\frac{v}{\zeta}+x-\bar{v} \zeta\right)^{2} .
$$

By matching the coefficients of (5.20) and (5.35), one readily obtains

$$
z=v^{2}, \quad w=2 x v, \quad y=x^{2}-2 v \bar{v},
$$

from which it follows that

$$
y_{+}=\frac{x^{2}+4 v \bar{v}}{3}, \quad y_{-}=\frac{x^{2}-8 v \bar{v}}{3}, \quad w_{+}=0, \quad w_{-}=2 x .
$$

Moreover, plugging into (5.22), one finds

$$
g_{2}=\frac{1}{3}\left(x^{2}+4 v \bar{v}\right)^{2}, \quad g_{3}=-\frac{2}{27}\left(x^{2}+4 v \bar{v}\right)^{3} .
$$


These last two equations are consistent with the limit $\tau \rightarrow 0$ of (5.29) computed using $E_{4}(\tau) \sim\left(\tau^{\prime}\right)^{4}, E_{6}(\tau) \sim\left(\tau^{\prime}\right)^{6}$,

$$
g_{2}=\frac{1}{3}\left(\frac{\pi \tau^{\prime}}{2 \omega}\right)^{4}\left(1+240 q^{\prime 2}+\cdots\right), \quad g_{3}=\frac{2}{27}\left(\frac{\pi \tau^{\prime}}{2 \omega}\right)^{6}\left(1-504 q^{2}+\cdots\right),
$$

provided we identify

$$
r \equiv \sqrt{x^{2}+4 v \bar{v}}=\frac{\pi \tau^{\prime}}{2 i \omega} .
$$

Using the fact that $\hat{E}_{2}(\tau)=E_{2}(\tau)-3 /\left(\pi \tau_{2}\right)$ is modular invariant of weight 2 , one obtains the asymptotic behavior of $\eta$ in (5.34),

$$
\eta(\tau) \sim \frac{1}{3} \omega\left(\frac{\pi \tau^{\prime}}{2 \omega}\right)^{2}\left(1-\frac{6 \mathrm{i}}{\pi \tau^{\prime}}-24 q^{\prime 2}\right) \sim-\frac{1}{3} \omega r^{2}+r+\cdots
$$

Thus, the Kähler potential reduces to

$$
K=4 \eta-\left(y_{+}+y_{-}\right) \omega=-2 \omega\left(x^{2}+2 v \bar{v}\right)+4 r+\cdots,
$$

in agreement with (5.2), up to an overall factor of 4, provided we set $c=\omega / 2$.

It remains to express $x, v, \bar{v}$ in terms of the complex coordinates $U, \bar{U}, Z, \bar{Z}$. Since $Z=v^{2}$, the only non-trivial task is to express $x$ in terms of $U, \bar{U}, Z, \bar{Z}$. Expanding $\mathcal{P}(v), \pi(v)$ on the axis $u \in \omega \mathbb{R}+\omega^{\prime}$ (i.e. $v \in \frac{\pi}{2} \tau+\mathbb{R}$ ) leads to

$$
\pi\left(y_{-}\right) \equiv \pi\left(v_{-}\right)=-\omega x+\operatorname{arctanh}(x / r)+\cdots
$$

which reproduces the condition (5.3) provided one identifies $U=u, \bar{U}=\bar{u}$,

$$
\frac{U+\bar{U}}{2}=\pi\left(y_{-}\right)=-\omega x+\operatorname{arcsinh}(x / \sqrt{4 v \bar{v}})+\cdots
$$

On the other hand, expanding the Weierstrass function and the elliptic integral $\pi$ on the axis $u \in \omega+\mathbb{R} \omega^{\prime}$ (i.e. $v \in \frac{\pi}{2}+\mathrm{i} \mathbb{R}$ ) and neglecting exponential corrections, the condition $\mathcal{P}\left(v_{+}\right)=y_{+}$is trivially satisfied independently of $u_{+}$, and $v_{+}$and related to $U-\bar{U}$ via

$$
\frac{U-\bar{U}}{2}=\pi\left(y_{+}\right) \equiv \pi\left(v_{+}\right)=-\mathrm{i} \tau^{\prime}\left(v_{+}-\frac{\pi}{2}\right) .
$$

The fact that the Kähler potential (5.42) depends only on $Z, \bar{Z}, U+\bar{U}$ is of course a consequence of the triholomorphic isometry recovered in the Taub-NUT limit. Utilizing the explicit expressions for the AH metric obtained in [29], one can also verify that in the strict Taub-Nut-limit one exactly recovers (5.4), provided $U=$ $u_{T N}, Z=v_{T N}, c=\omega / 2$. 


\subsubsection{Exponential deviations from the Taub-NUT limit}

Let us now incorporate the first exponential correction to the Taub-NUT limit. Our aim is to determine the twistor line $\eta^{(4)}(\zeta)$ as a function of the complex coordinates $U, \bar{U}, Z, \bar{Z}$, to first order in $q^{\prime}$. We thus deform the relations (5.36) into

$$
w=2 x v+q^{\prime} \delta w+\cdots, \quad y=x^{2}-2 v \bar{v}+q^{\prime} \delta y+\cdots,
$$

keeping the relation $z=v^{2}$ undeformed (indeed, $Z=2 \sqrt{z}$ is an exact relation, and we work at fixed $U, \bar{U}, Z, \bar{Z}$.) Computing $y_{ \pm}$and $w_{ \pm}$from (5.46), inserting into (5.22) and comparing to (5.39), we learn that the relation (5.40) is deformed to

$$
\tau^{\prime}=\frac{2 i \omega r}{\pi}+\frac{i \omega}{\pi r^{3}}\left[\delta y\left(x^{2}-2 v \bar{v}\right)+3 x \Delta\right] q^{\prime}+\cdots,
$$

where $\Delta \equiv v \delta \bar{w}+\bar{v} \delta w$. Note that we define $r$ to be equal to $\sqrt{x^{2}+4 v \bar{v}}$, and $x$ to be the function of $U, \bar{U}, Z, \bar{Z}$ given implicitly by (5.44). We also expand the uniformizing parameters $v_{ \pm}$associated to $y_{ \pm}$as

$$
v_{-}=-\frac{\pi}{2} \tau+\frac{\pi}{2 \omega r} \operatorname{arcsinh}(x / \sqrt{4 v \bar{v}})+q^{\prime} \delta v_{-}, \quad v_{+}=\frac{\pi}{4 \omega r}(U-\bar{U})+q^{\prime} \delta v_{+} .
$$

The two conditions $\mathcal{P}\left(v_{+}\right)=y_{+}$and $\mathcal{P}\left(v_{-}\right)=y_{-}$may be used to compute $\delta y$ and $\delta v_{-}$, respectively,

$$
\begin{aligned}
\delta y= & \frac{8 r^{4} \cosh (U-\bar{U})+x \Delta}{2 v \bar{v}} \\
\delta v_{-}= & \frac{\pi}{8 \omega v \bar{v} r^{3}}\left[r \Delta+12 x r^{3} \cosh (U-\bar{U})-\right. \\
& \left.-\left(x \Delta+8 r^{2}\left(x^{2}-2 v \bar{v}\right) \cosh (U-\bar{U})\right) \operatorname{arcsinh}(x / \sqrt{4 v \bar{v}})\right] .
\end{aligned}
$$

Finally, the conditions that $\pi\left(v_{+}\right)$and $\pi\left(v_{-}\right)$have no variation (because $U, \bar{U}$ are fixed) allows to compute $\delta v_{+}$and $\Delta$ :

$$
\begin{aligned}
\Delta & =-4 r^{2} x \frac{3-2 \omega r}{1-\omega r} \cosh (U-\bar{U}), \\
\delta v_{+} & =-2 \pi \sinh (U-\bar{U})-\frac{\pi}{4 \omega} \frac{x^{4}-16 v^{2} \bar{v}^{2}-4 \omega r^{3} v \bar{v}+8 r^{2} v \bar{v}}{r^{3} v \bar{v}(1-\omega r)}(U-\bar{U}) \cosh (U-\bar{U}) .
\end{aligned}
$$

While these relations are not sufficient to fix $\delta w$ and $\delta \bar{w}$ separately, they are sufficient to obtain the leading exponential correction to the Kähler potential (5.42),

$$
K=-2 \omega\left(x^{2}+2 v \bar{v}\right)+4 r-\frac{4 r^{3}}{v \bar{v}} \cosh (U-\bar{U}) q^{\prime}+\mathcal{O}\left(q^{\prime 2}\right) .
$$


Using the same spherical coordinates as for Taub-NUT space (5.5), this may be rewritten as

$$
K=-\frac{1}{2} \omega r^{2}(3+\cos 2 \theta)+4 r-16 \frac{\cos 2 \psi}{(\sin \theta)^{2}} r \mathrm{e}^{-2 \omega r}+\mathcal{O}\left(q^{\prime 2}\right) .
$$

The variation of $K$ may be rewritten as in (4.27) as the Penrose transform of the holomorphic function

$$
H_{(1)}\left(\eta, \mu^{[\infty]}, \zeta\right)=4 \eta \cos \left(4 \mu^{[\infty]}\right),
$$

where $\eta$ and $\mu^{[\infty]}$ are the global sections of unperturbed Taub-NUT space, given in (5.9). Indeed, an explicit computation shows that

$$
\oint_{C} \frac{\mathrm{d} \zeta}{2 \pi \mathrm{i} \zeta} H_{(1)}=-16 \frac{\cos (2 \psi)}{(\sin \theta)^{2}} r \mathrm{e}^{-2 \omega r},
$$

where $C$ denotes the figure-eight contour enclosing the two poles $\zeta_{+}=\mathrm{e}^{\mathrm{i} \phi} \cot (\theta / 2)$ and $\zeta_{-}=-\mathrm{e}^{\mathrm{i} \phi} \tan (\theta / 2)$ clockwise and counterclockwise, respectively.

The main result of this section (5.55) provides a remarkably simple and elegant way to summarize the deviation of the AH manifold away from its Taub-NUT limit at leading order. It should be noted that the exponential dependence of $H_{(1)}$ on $\mu^{[\infty]}$ is fixed by the requirement that instanton corrections preserve discrete shifts $\varrho \rightarrow \varrho+\pi$. Instanton corrections to the metric components have been analyzed in [25]. It would be interesting to extend this analysis to other HK spaces whose metric is known only implicitly, such as the moduli space of monopoles of higher charge $[8,28]$, or Dancer's manifold $[15,14]$.

\section{Acknowledgements}

We are very grateful to R. Ionas for correspondence on [29], M. Roček for informing us of his upcoming work [33], and to A. Neitzke for enlightening discussions. The research of S. Alexandrov is supported by CNRS and by the contract ANR-05-BLAN-0029-01. The research of B. Pioline is supported in part by ANR(CNRS-USAR) contract no. 05-BLAN-0079-01. F. Saueressig acknowledges financial support from the ANR grant BLAN06-3-137168. S. Vandoren thanks the Federation de Recherches "Interactions Fondamentales" and LPTHE at Jussieu for hospitality and financial support. Part of this work is also supported by the EU-RTN network MRTN-CT-2004-005104 "Constituents, Fundamental Forces and Symmetries of the Universe".

Open Access This article is distributed under the terms of the Creative Commons Attribution Noncommercial License which permits any noncommercial use, distribution, and reproduction in any medium, provided the original author(s) and source are credited. 


\section{Appendix A: Direct Verification of the HK Property}

In this appendix, we give a direct verification that the complex manifold with Kähler potential (4.26) is $\mathrm{HK}$, to first order in the perturbation.

We first need some identities for the function $G_{I}$ defined in (4.13). Substituting the result (4.12) for $\hat{v}_{[i]}^{I}$ and using the relations

$$
\begin{aligned}
H_{(1)}^{[i j]}{ }_{I} & =\partial_{x^{I}} H_{(1)}^{[i j]}+\mathrm{i} H_{(1)}^{[i j] J}\left(2 \partial_{x^{I}} \breve{\mu}_{J}^{[j]}-\left(H_{I J}^{[j 0]}+H_{I J}^{[j \infty]}\right)\right)= \\
& =\zeta \partial_{v^{I}} H_{(1)}^{[i j]}+\mathrm{i} H_{(1)}^{[i j] J}\left(2 \zeta \partial_{v^{I}} \breve{\mu}_{J}^{[j]}-\left(H_{I J}^{[j 0]}+H_{I J}^{[j \infty]}\right)\right),
\end{aligned}
$$

the function (4.13) can be written in one of the two following forms

$$
\begin{aligned}
G_{I}= & \partial_{x^{I}} H_{(1)}^{[i j]}-2 \mathrm{i} H_{(1)}^{[i j] J} H_{I J}^{[i j]}+ \\
& +\mathrm{i} \sum_{k} \oint_{C_{k}} \frac{\mathrm{d} \zeta^{\prime}}{2 \pi \mathrm{i} \zeta^{\prime}}\left(\frac{\zeta^{3}+\zeta^{\prime 3}}{\zeta \zeta^{\prime}\left(\zeta^{\prime}-\zeta\right)} H_{(1)}^{[i k] J}\left(\zeta^{\prime}\right) H_{I J}^{[i j]}(\zeta)+\frac{\zeta+\zeta^{\prime}}{\zeta^{\prime}-\zeta} H_{(1)}^{[i j] J}(\zeta) H_{I J}^{[i k]}\left(\zeta^{\prime}\right)\right) \\
= & \zeta \partial_{v^{I}} H_{(1)}^{[i j]}-2 i H_{(1)}^{[i j] J} H_{I J}^{[i j]}+ \\
& +\mathrm{i} \sum_{k} \oint_{C_{k}} \frac{\mathrm{d} \zeta^{\prime}}{2 \pi \mathrm{i} \zeta^{\prime 2}}\left(\frac{\zeta^{3}+\zeta^{\prime 3}}{\zeta\left(\zeta^{\prime}-\zeta\right)} H_{(1)}^{[i k] J}\left(\zeta^{\prime}\right) H_{I J}^{[i j]}(\zeta)+\frac{\zeta\left(\zeta+\zeta^{\prime}\right)}{\zeta^{\prime}-\zeta} H_{(1)}^{[i j] J}(\zeta) H_{I J}^{[i k]}\left(\zeta^{\prime}\right)\right),
\end{aligned}
$$

where $\zeta$ is inside of $C_{j}$. These representations can be used to obtain

$$
\begin{aligned}
\oint_{C} \frac{\mathrm{d} \zeta}{2 \pi \mathrm{i} \zeta} G_{I}= & \partial_{x^{I}} \oint_{C} \frac{\mathrm{d} \zeta}{2 \pi \mathrm{i} \zeta} H_{(1)}- \\
& -\mathrm{i}\left(\oint_{C} \frac{\mathrm{d} \zeta}{2 \pi \mathrm{i} \zeta^{2}} H_{(1)}^{J} \oint_{C} \frac{\mathrm{d} \zeta}{2 \pi \mathrm{i}} H_{I J}-\oint_{C} \frac{\mathrm{d} \zeta}{2 \pi \mathrm{i}} H_{(1)}^{J} \oint_{C} \frac{\mathrm{d} \zeta}{2 \pi \mathrm{i} \zeta^{2}} H_{I J}\right) \\
\oint_{C} \frac{\mathrm{d} \zeta}{2 \pi \mathrm{i} \zeta^{2}} G_{I}= & \partial_{v^{I}} \oint_{C} \frac{\mathrm{d} \zeta}{2 \pi \mathrm{i} \zeta} H_{(1)}- \\
& -\mathrm{i}\left(\oint_{C} \frac{\mathrm{d} \zeta}{2 \pi \mathrm{i} \zeta^{2}} H_{(1)}^{J} \oint_{C} \frac{\mathrm{d} \zeta}{2 \pi \mathrm{i} \zeta} H_{I J}-\oint_{C} \frac{\mathrm{d} \zeta}{2 \pi \mathrm{i}} H_{(1)}^{J} \oint_{C} \frac{\mathrm{d} \zeta}{2 \pi \mathrm{i} \zeta^{3}} H_{I J}\right)
\end{aligned}
$$

where the sum over contours and the patch indices are suppressed. These results may be used to check the integrability condition for the existence of the Kähler potential,

$$
\partial_{w_{I}}\left(\mathcal{L}_{v^{J}}+\hat{v}_{0}^{K} \mathcal{L}_{v^{K} v^{J}}+\overline{\hat{v}}_{0}^{K} \mathcal{L}_{\bar{v}^{K} v^{J}}\right)=-\partial_{u^{J}}\left(x^{I}+\mathrm{i} \mathcal{L}_{\varrho_{I}}\right) .
$$

Since the Lagrangian $\mathcal{L}$ is an integral of a holomorphic function of only $2 d+1$ variables, it must satisfy some relations. Indeed, it may be checked explicitly that 


$$
\begin{aligned}
\mathcal{L}_{x^{I} x^{J}}+\mathcal{L}_{u^{I} \bar{u}^{J}=} & -\mathrm{i}\left(\mathcal{L}_{x^{I} x^{K}} \mathcal{L}_{\varrho_{K} x^{J}}-\mathcal{L}_{x^{I} \varrho_{K}} \mathcal{L}_{x^{K} x^{J}}+\mathcal{L}_{u^{I} \varrho_{K}} \mathcal{L}_{x^{K} \bar{u}^{J}}-\mathcal{L}_{u^{I} x^{K}} \mathcal{L}_{\varrho_{K} \bar{u}^{J}}\right)- \\
& -\left(\mathcal{L}_{x^{I} x^{K}} \mathcal{L}_{x^{L} x^{J}}+\mathcal{L}_{u^{I} x^{K}} \mathcal{L}_{x^{L} \bar{u}^{J}}\right) \mathcal{L}_{\varrho_{K} \varrho_{L}}, \\
\mathcal{L}_{x^{I} u^{J}}-\mathcal{L}_{u^{I} x^{J}}= & -\mathrm{i}\left(\mathcal{L}_{u^{I} x^{K}} \mathcal{L}_{\varrho_{K} x^{J}}-\mathcal{L}_{x^{I} \varrho_{K}} \mathcal{L}_{x^{K} u^{J}}+\mathcal{L}_{x^{I} x^{K}} \mathcal{L}_{\varrho_{K} u^{J}}-\mathcal{L}_{u^{I} \varrho_{K}} \mathcal{L}_{x^{K} x^{J}}\right)- \\
& -\left(\mathcal{L}_{x^{I} x^{K}} \mathcal{L}_{x^{L} u^{J}}-\mathcal{L}_{u^{I} x^{K}} \mathcal{L}_{x^{L} x^{J}}\right) \mathcal{L}_{\varrho_{K} \varrho_{L}} .
\end{aligned}
$$

which generalize the standard identities for the unperturbed case,

$$
\mathcal{L}_{(0) x^{I} x^{J}}+\mathcal{L}_{(0) v^{I} \bar{v}^{J}}=0, \quad \mathcal{L}_{(0) x^{I} v^{J}}-\mathcal{L}_{(0) v^{I} x^{J}}=0 .
$$

Equivalently, (A.6) and (A.7) may be rewritten as

$$
\begin{aligned}
& \left(\partial_{x^{I}}+\mathrm{i} \mathcal{L}_{x^{I} x^{K}} \partial_{\varrho_{K}}\right)\left(\partial_{x^{J}}-\mathrm{i} \mathcal{L}_{x^{J} x^{L}} \partial_{\varrho_{L}}\right) \mathcal{L}+ \\
& \quad+\left(\partial_{u^{I}}-\mathrm{i} \mathcal{L}_{u^{I} x^{K}} \partial_{\varrho_{K}}\right)\left(\partial_{\bar{u}^{J}}+\mathrm{i} \mathcal{L}_{\bar{u}^{J} x^{L}} \partial_{\varrho_{L}}\right) \mathcal{L}=0, \\
& \left(\partial_{x^{I}}+\mathrm{i} \mathcal{L}_{x^{I} x^{K}} \partial_{\varrho_{K}}\right)\left(\partial_{u^{J}}-\mathrm{i} \mathcal{L}_{u^{J} x^{L}} \partial_{\varrho_{L}}\right) \mathcal{L}+ \\
& \quad-\left(\partial_{x^{J}}+\mathrm{i} \mathcal{L}_{x^{J} x^{K}} \partial_{\varrho_{K}}\right)\left(\partial_{u^{I}}-\mathrm{i} \mathcal{L}_{u^{I} x^{L}} \partial_{\varrho_{L}}\right) \mathcal{L}=0 .
\end{aligned}
$$

These properties can be used to compute the inverse metric,

$$
\begin{aligned}
K^{\bar{u}^{I} u^{J}} & =-\mathcal{L}^{x^{I} x^{J}}+\mathcal{L}_{\varrho_{I} \varrho_{J}}-\mathrm{i}\left[\mathcal{L}^{x^{I} x^{K}} \mathcal{L}_{x^{K} \varrho_{J}}-\mathcal{L}_{\varrho_{I} x^{K}} \mathcal{L}^{x^{K} x^{J}}\right], \\
K^{\bar{u}^{I} w_{J}} & =-\mathcal{L}^{x^{I} x^{K}} \mathcal{L}_{x^{K} u^{J}}-\mathrm{i}\left[\mathcal{L}_{\varrho_{I} u^{J}}-\mathcal{L}_{\varrho_{I} x^{K}} \mathcal{L}^{x^{K} x^{L}} \mathcal{L}_{x^{L} u^{J}}\right], \\
K^{\bar{w}_{I} u^{J}} & =-\mathcal{L}_{\bar{u}^{I} x^{K}} \mathcal{L}^{x^{K} x^{J}}+\mathrm{i}\left[\mathcal{L}_{\bar{u}^{I} \varrho_{J}}-\mathcal{L}_{\bar{u}^{I} x^{M}} \mathcal{L}^{x^{M} x^{N}} \mathcal{L}_{x^{N} \varrho_{J}}\right], \\
K^{\bar{w}_{I} w_{J}} & =\mathcal{L}_{\bar{u}^{I} u^{J}}-\mathcal{L}_{\bar{u}^{I} x^{K}} \mathcal{L}^{x^{K} x^{L}} \mathcal{L}_{x^{L} u^{J}} .
\end{aligned}
$$

Recalling that $\omega^{+}=\mathrm{d} w_{I} \wedge \mathrm{d} u^{I}$, it is now straightforward to check that the complex structures satisfy the algebra of the quaternions, e.g.

$$
J^{+} J^{-}=-\frac{1}{2}\left(\mathbf{1}+\mathrm{i} J^{3}\right) \leftrightarrow\left(\omega^{+}\right)_{\alpha \gamma} K^{\gamma \bar{\delta}}\left(\omega^{-}\right)_{\bar{\delta} \bar{\beta}}=K_{\alpha \bar{\beta}} .
$$

\section{References}

1. Alexandrov, S., Saueressig, F., Vandoren, S.: Membrane and fivebrane instantons from quaternionic geometry. JHEP 09, 040 (2006) (hep-th/0606259)

2. Alexandrov, S.: Quantum covariant c-map. JHEP 05, 094 (2007) (hep-th/0702203)

3. Alexandrov, S., Pioline, B., Saueressig, F., Vandoren, S.: Linear perturbations of quaternionic metrics $(0810.1675)$

4. Alexandrov, S., Pioline, B., Saueressig, F., Vandoren, S.: D-instantons and twistors (0812.4219)

5. Alvarez-Gaume, L., Freedman, D.Z.: Ricci flat kahler manifolds and supersymmetry. Phys. Lett. B94, 171 (1980)

6. Aspinwall, P.S.: Aspects of the hypermultiplet moduli space in string duality. JHEP 04, 019 (1998) (hep-th/9802194)

7. Atiyah, M.F., Hitchin, N.J., Drinfeld, V., Manin, Y.: Construction of instantons. Phys. Lett. A65, 185-187 (1978) 
8. Atiyah, M.F., Hitchin, N.J.: The Geometry and Dynamics of Magnetic Monopoles. M.B. Porter Lectures, Princeton Univ. Press, New Jersey (1988)

9. Bagger, J, Witten, E.: Matter couplings in $\mathcal{N}=2$ supergravity. Nucl. Phys. B222, 1 (1983)

10. Becker, K., Becker, M., Strominger, A.: Five-branes, membranes and nonperturbative string theory. Nucl. Phys. B456, 130-152 (1995) (hep-th/9507158)

11. Besse, A.: Einstein Manifolds. Springer, Heidelberg (1987)

12. Bielawski, R.: Twistor quotients of hyperkahler manifolds (math.DG/0006142)

13. Cecotti, S., Ferrara, S., Girardello, L.: Geometry of type II superstrings and the moduli of superconformal field theories. Int. J. Mod. Phys. A4, 2475 (1989)

14. Chalmers, G.: The implicit metric on a deformation of the Atiyah-Hitchin manifold. Phys. Rev. D58, 125011 (1998) (hep-th/9709082)

15. Dancer, A.: A family of hyper-Kähler manifolds. Quart. J. Math. Oxf. 45, 463 (1994)

16. de Wit, B., Roček, M.: Improved tensor multiplets. Phys. Lett. B109, 439 (1982)

17. de Wit, B., Roček, M., Vandoren, S.: Hypermultiplets, hyperkähler cones and quaternion-Kähler geometry. JHEP 02, 039 (2001) (hep-th/0101161)

18. Dorey, N., Khoze, V.V., Mattis, M.P., Tong, D., Vandoren, S.: Instantons, three-dimensional gauge theory, and the Atiyah-Hitchin manifold. Nucl. Phys. B502, 59-93 (1997) (hep-th/9703228)

19. Dorey, N., Tong, D., Vandoren, S.: Instanton effects in three-dimensional supersymmetric gauge theories with matter. JHEP 04, 005 (1998) (hep-th/9803065)

20. Dunajski, M., Mason, L.: Twistor theory of hyper-Kähler metrics with hidden symmetries. J. Math. Phys. 44, 3430 (2003)

21. Ferrara, S., Sabharwal, S.: Quaternionic manifolds for type II superstring vacua of Calabi-Yau spaces. Nucl. Phys. B332, 317 (1990)

22. Fraser, C., Tong, D.: Instantons, three dimensional gauge theories and monopole moduli spaces. Phys. Rev. D58, 085001 (1998) (hep-th/9710098)

23. Gibbons, G.W., Manton, N.S.: The Moduli space metric for well separated BPS monopoles. Phys. Lett. B356, 32-38 (1995) (hep-th/9506052)

24. Günaydin, M., Neitzke, A., Pioline, B.: Topological wave functions and heat equations. JHEP 12, 070 (2006) (hep-th/0607200)

25. Hanany, A., Pioline, B.: (Anti-)instantons and the Atiyah-Hitchin manifold. JHEP 07, 001 (2000) (hep-th/0005160)

26. Hitchin, N.J., Karlhede, A., Lindström, U., Roček, M.: Hyperkähler metrics and supersymmetry. Commun. Math. Phys. 108, 535 (1987)

27. Hitchin, N.J.: The self-duality equations on a Riemann surface. Proc. Lond. Math. Soc. 55, 59-126 (1987)

28. Houghton, C.J.: On the generalized Legendre transform and monopole metrics. JHEP 02, 042 (2000) (hep-th/9910212)

29. Ionas, R.A.: Elliptic constructions of hyperkaehler metrics I: the Atiyah-Hitchin manifold (arXiv:0712.3598 [math.DG])

30. Ivanov I.T., Roček, M.: Supersymmetric sigma models, twistors, and the AtiyahHitchin metric. Commun. Math. Phys. 182, 291-302 (1996) (hep-th/9512075)

31. Karlhede, A., Lindström, U., Roček, M.: Selfinteracting tensor multiplets in $\mathcal{N}=2$ superspace. Phys. Lett. B147, 297 (1984)

32. Lindström, U., Roček, M.: New hyperkähler metrics and new supermultiplets. Commun. Math. Phys. 115, 21 (1988)

33. Lindström, U., Roček, M.: Properties of hyperkähler manifolds and their twistor spaces (arXiv:0807.1366 [hep-th])

34. MacCallum, M.A.H., Penrose, R.: Twistor theory: an approach to the quantization of fields and space-time. Phys. Rept. 6, 241-316 (1972) 
35. Ooguri, H., Vafa, C.: Two-dimensional black hole and singularities of CY manifolds. Nucl. Phys. B463, 55-72 (1996) (hep-th/9511164)

36. Robles-Llana, D., Saueressig, F., Vandoren, S.: String loop corrected hypermultiplet moduli spaces. JHEP 03, 081 (2006) (hep-th/0602164)

37. Robles-Llana, D., Roček, M., Saueressig, F., Theis, U., Vandoren, S.: Nonperturbative corrections to 4D string theory effective actions from $\operatorname{SL}(2, \mathrm{Z})$ duality and supersymmetry. Phys. Rev. Lett. 98, 211602 (2007) (hep-th/0612027)

38. Robles-Llana, D., Saueressig, F., Theis, U., Vandoren, S.: Membrane instantons from mirror symmetry (arXiv:0707.0838 [hep-th])

39. Salamon, S.M.: Quaternionic Kähler manifolds. Invent. Math. 67, 143-171 (1982)

40. Salamon, S.M.: Differential geometry of quaternionic manifolds. Ann. Sci. Ecol. Norm. Super. 19, 31-55 (1986)

41. Saueressig, F., Vandoren, S.: Conifold singularities, resumming instantons and nonperturbative mirror symmetry. JHEP 07, 018 (2007) (arXiv:0704.2229 [hep-th])

42. Seiberg, N., Witten, E.: Gauge dynamics and compactification to three dimensions (hep-th/9607163)

43. Swann, A.: Hyper-Kähler and quaternionic Kähler geometry. Math. Ann. 289, 421-450 (1991)

44. Witten, E.: Heterotic string conformal field theory and A-D-E singularities. JHEP 02, 025 (2000) (hep-th/9909229) 